\title{
The influence of the outer bath in the dynamics of axisymmetric liquid bridges
}

\author{
By ANGEL SANZ \\ Laboratorio de Aerodinámica, E.T.S.I. Aeronáuticos, Universidad Politécnica, \\ 28040 Madrid, Spain
}

(Received 12 April 1984 and in revised form 11 January 1985)

The main effects on the dynamies of a liquid bridge due to the presence of an outer liquid, as occur in experiments using the Plateau-tank technique, are considered. The one-dimensional nonlinear model developed here allows us to perform the computation of both breaking processes and oscillatory motions of slender liquid bridges, although in this paper only the results concerning breaking processes are reported. Additionally, the oscillatory motions are studied both experimentally and by using a new linear model. Results from both sources show good agreement.

\section{Introduction}

Recent availability of space experiments on fluid mechanics is giving rise to interest in liquid behaviour studies in microgravity conditions. One of the fluid configurations receiving increasing attention in these experiments is the liquid bridge. This is because of the various valuable uses to which it can be put (Provost \& Joyal 1972; Leybold-Heraeus 1977). Some theoretical models have already been developed for studying static behaviour (Gillette \& Dyson 1971; Martinez 1976), and dynamic response to purely mechanical disturbances (Da Riva \& Meseguer 1978; Harriot \& Brown 1983; Meseguer 1983a,b), prior to more complex investigations, taking thermal, electrical and other disturbances (Napolitano 1978; Da Riva \& Alvarez Pereira 1982) into account. An extensive, up-to-date review of the contributions to these subjects can be found in Meseguer \& Sanz (1985).

The expense and difficulty involved in performing space experiments is one of the reasons why intensive development of experimental support is carried out on Earth, so that in-flight experiments can be prepared as completely as possible beforehand. Each technique employed in performing on-Earth experiments has its own advantages, drawbacks and restrictions. One of these techniques is neutral buoyancy, the so-called Plateau-tank simulation, in which the liquid bridge is surrounded by an outer liquid (bath) to compensate for the hydrostatic pressure along the interface. This technique has been chosen several times by experimenters (Mason 1970; Erle, Gillette \& Dyson 1970; Carruthers \& Grasso 1972; Coriell, Hardy \& Cordes 1977; Sanz 1983; Sanz \& Martinez 1983), for its simplicity and low demand on highly sophisticated and expensive equipment, amongst other reasons. However, in most cases, when experimental and theoretical results are compared, the bath influence has not been taken into account; this is valid in static phenomena but not completely applicable when dynamic processes are involved.

On the other hand, in the above-mentioned theoretical models the liquid bridge should be surrounded by a vacuum or a gas with far lower density than the liquid, an assumption which is not applicable to neutral-buoyancy experiments. Although 
some models dealing with a liquid bridge surrounded by an outer fluid can be found in linear-jet-stability theory (Levich 1962; Phinney 1973), they are not relevant to this study because they only retain outer fluid convective terms, ignoring inertial ones, hence not giving a suitable estimation of surrounded-liquid-bridge dynamics. Other studies (Tomotika 1935; Bauer 1982), based also on the jet theory, but including the appropriate terms, deal with resonant frequencies and linear stability of infinite surrounded liquid columns. In order to compare the results from the above-mentioned models with those of finite liquid bridges, these authors should assume that the disks are placed in zero-axial-velocity sections of the jet or column, but this implies that the three-phase contact line can move freely over the disks, since the jet theory is unable to fulfil both the zero-normal-velocity and anchored-interface conditions in the same section. On the contrary, from an experimental point of view, an anchored contact line is far more realistic than a free one, and, according to Meseguer (1983a), the boundary conditions imposed strongly affect results: characteristic evolution times in the liquid-bridge problem are significantly higher than those obtained from the capillary-jet theory.

The aim of this paper is to analyse the influence of the outer liquid on the dynamics of the liquid bridge. There are three parts: first, the breaking process is studied by means of a nonlinear one-dimensional model; secondly, oscillation motions are analysed using a new linear three-dimensional model; finally, there is an experimental study of the resonant frequencies and results are compared with predictions obtained in the second part. Accounts of experiments on breaking processes can be found in Meseguer \& Sanz (1985).

In this paper, the densities of the inner and the outer liquids are assumed to be different, in order to find out the influence of the density ratio, so that a hypothesis of absence of body forces, like gravity, can be made to avoid the effect of hydrostatic pressure on the interface shape. Nevertheless, if both liquids have the same density, as in Plateau's configurations, hydrostatic pressure is balanced along the interface and the aforementioned hypothesis becomes unnecessary.

The effect of the viscosity on the liquid-bridge dynamics (oscillation motion and breaking) can be separated in two parts: inside the bulk liquid and at its interface. The effects in the bulk liquid are small in the experimental configurations more widely used in simulated microgravity, as demonstrated by Meseguer (1983a), and it seems reasonable to assume here that shear stresses at the interface are of the same order and thus negligible. However, to demonstrate that point, further work would be required.

\section{General equations for the axisymmetric liquid bridge}

Let us consider a liquid bridge held by surface-tension forces between two parallel, coaxial, equal-diameter solid disks, surrounded by an outer liquid immiscible with the inner one, and limited by two plane ring elements, prolongation of the disks, and by a circular-cross-section cylinder, coaxial with the liquid bridge, as shown in figure 1.

The problem to be solved concerns the evolution of the previously described configuration, under axisymmetric disturbances. In this paper the study is limited to processes in which the initial interface shape differs slightly from a cylinder.

To perform this study, widely used assumptions (Meseguer 1983 $a$ ) are introduced:

(a) movements inside both the liquid bridge and the bath are due only to capillary-pressure gradients generated by the deformation of the interface; 


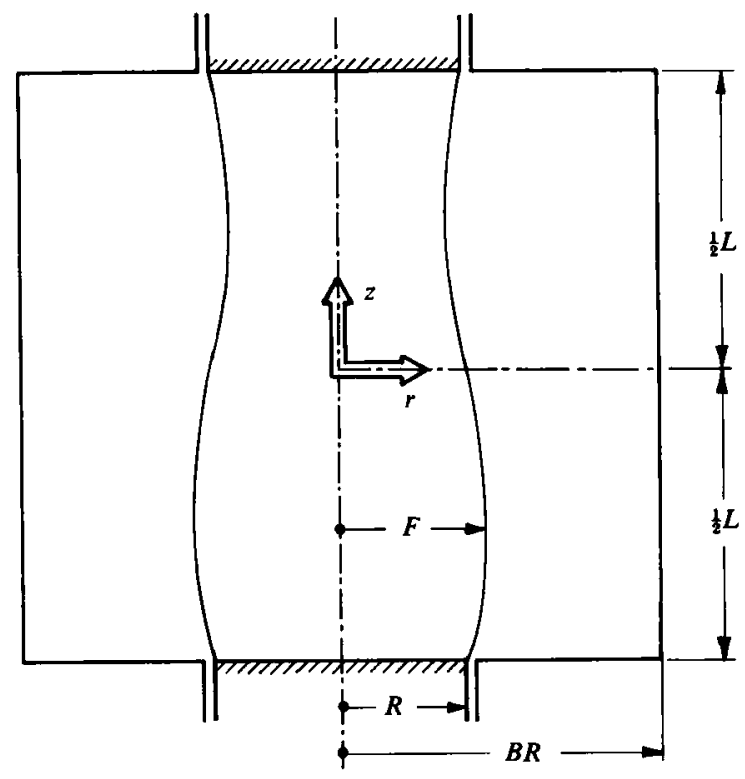

Figure 1. Geometry and coordinate system for the surrounded liquid bridge.

(b) as mentioned above, inertial forces due to a non-uniform displacement of the liquid bridge as a whole are absent;

(c) the problem is considered to be independent of the azimuthal coordinate, since we are only dealing with axisymmetric configurations and perturbations;

(d) the densities of both liquids, as well as interface properties (surface tension), are uniform and constant.

Under such assumptions the non-dimensional equations for the inviscid, axisymmetric, non-rotating flow in cylindrical coordinates are

$$
\begin{gathered}
U_{r}^{j}+\frac{U^{j}}{r}+W_{z}^{j}=0 \\
U_{t}^{j}+U^{j} U_{r}^{j}+W^{j} U_{z}^{j}=-\frac{P_{r}^{j} \rho^{i}}{\rho^{j}}, \\
W_{t}^{j}+U^{j} W_{r}^{j}+W^{j} W_{z}^{j}=-\frac{P_{z}^{j} \rho^{i}}{\rho^{j}},
\end{gathered}
$$

where $U^{j}, W^{j}$ and $P^{j}$ represent the non-dimensional radial and axial velocities and pressure respectively, the superscript $j$ refers to the different liquids: ' $i$ ', liquid bridge, and ' $o$ ', bath, and the subscripts $t, r$ and $z$ mean differentiation with respect to time, radial or axial coordinates respectively. In these expressions, lengths have been made dimensionless with disk radius $R$, velocities with $\left(\sigma / \rho^{1} R\right)^{\frac{1}{2}}$ (where $\sigma$ is the interface tension between the two liquids and $\rho^{1}$ the density of the inner liquid), time with $\left(\rho^{1} R^{3} / \sigma\right)^{\frac{1}{2}}$ and reduced gauge pressure $\left(\mathscr{P}^{1}-\mathscr{P}^{r}\right) / \rho^{1}$ with $\sigma / \rho^{1} R$, where $\mathscr{P}^{\mathrm{i}}$ is the pressure at each point and $\mathscr{P}$ r a reference pressure.

The boundary conditions at the solid surfaces are

$$
\begin{aligned}
W^{\prime}(r, \pm A, t) & =0, \\
U^{\circ}(B, z, t) & =0,
\end{aligned}
$$


$A=L / 2 R$ being the slenderness of the liquid bridge and $B$ the dimensionless radius of the external cylindrical container (wall position). At the axis of symmetry

$$
\begin{aligned}
& U^{\mathrm{i}}(0, z, t)=0, \\
& W_{r}^{\mathrm{i}}(0, z, t)=0 .
\end{aligned}
$$

At the interface, $r=F(z, t)$, two conditions hold. The first expresses the normal force balance between the capillary pressure and the pressure jump across the interface. In dimensionless form,

$$
P^{1}-P^{0}=\frac{\left(1+F_{z}^{2}\right) / F-F_{z z}}{\left(1+F_{z}^{2}\right)^{\frac{3}{2}}}
$$

The second condition, stating that the interface is a material surface, that is, the mass-flow rate through it is zero, yields

$$
F_{t}-U^{j}+W^{j} F_{z}=0
$$

The interface has its own conditions, showing that the contact line remains anchored to the disk edges,

$$
F( \pm A, t)=1 \text {, }
$$

and that the volume enclosed remains the same, in this case the volume of a cylinder,

$$
\int_{-A}^{A} F^{2}(z, t) \mathrm{d} z=2 \Lambda .
$$

The formulation of the problem is completed by the setting of suitable initial conditions, i.e.

$$
F(z, 0)=F_{0}(z), \quad W^{j}(r, z, 0)=U^{j}(r, z, 0)=0 .
$$

The complexity of the system seems not to allow of an analytical solution, one of the main problems rising from the anchoring condition. If this condition is removed, the submerged-jet configuration would be brought about.

\section{One-dimensional inviscid slice (ODIS) model}

To solve the surrounded-liquid-bridge problem, a one-dimensional inviscid slice (ODIS) model, already used in isolated cylindrical and non-cylindrical liquid-bridgebreaking processes, both in zero-gravity and small-axial-gravity conditions (Meseguer 1983a,b; Meseguer \& Sanz 1985; Meseguer, Sanz \& Rivas 1983; Rivas \& Meseguer 1984), has been employed. General considerations on numerical and asymptotic studies, validity of the solutions, etc. can be found in these papers. Only specific differences of surrounded problem are treated here.

In this paragraph, the applicability conditions of the one-dimensional model to the surrounded-liquid-bridge problem are deduced. The influence of the outer liquid is discussed through a linear analysis. A numerical integration of the nonlinear equations is made, and the results obtained for the breaking process are presented, showing the quantitative influence of the outer liquid on global quantities which can easily be experimentally measured (such as breaking time and volume of drops resulting from breaking). Finally, some considerations on nonlinear asymptotic analysis are made.

\subsection{One-dimensional hypothesis}

The one-dimensional hypothesis consists in assuming axial velocity as independent of the radial coordinate. Its suitability for the study of the stability and the breaking 
process of non-surrounded slender liquid bridges has been deduced by Meseguer $(1983 a)$ by using an order-of-magnitude analysis. Results (Meseguer et al. 1983; Meseguer \& Sanz 1985) show that it is valid at least for $A>2$. A similar approach can be used to obtain conditions of applicability for surrounded liquid bridges. Let $U_{\mathrm{c}}^{\mathrm{o}}$ and $W_{\mathrm{c}}^{\mathrm{o}}$ respectively represent radial and axial characteristic velocities of the outer liquid; then $U_{\mathrm{c}}^{\mathrm{o}} \sim W_{\mathrm{c}}^{\mathrm{o}}(B-1) R / L$ can be deduced from the continuity equation (2.1). Since we are dealing with an irrotational flow, $\nabla \times V=0$; then

$$
\frac{U_{\mathrm{c}}^{\mathrm{o}}}{L} \sim \frac{\Delta_{r} W_{\mathrm{c}}^{\mathrm{o}}}{(B-1) R},
$$

where $\Delta_{r} W_{\mathrm{c}}^{\mathrm{o}}$ stands for the characteristic radial variation of the axial velocity. Thus the ratio of the convective terms appearing in (2.3) is

$$
\frac{U^{0} W_{r}^{\mathrm{o}}}{W^{\mathrm{o}} W_{z}^{\mathrm{o}}} \sim\left|\frac{(B-1) R}{L}\right|^{2}=\left|\frac{B-1}{2 A}\right|^{2} \ll 1 .
$$

For the case we are interested in, the breaking process of slender liquid bridges (in which $A \sim \pi$ ), this inequality is satisfied if $B-1=O(1)$. The condition for the inner flow could be found replacing $(B-1) R$ by inner characteristic radial length $R$, yielding

$$
\frac{U^{1} W_{r}^{\mathrm{i}}}{W^{1} W_{z}^{1}} \sim \frac{1}{4 \Lambda^{2}} \ll 1 .
$$

In consequence, according to (3.2) and (3.3) the term that contains $U^{j}$ can be eliminated in (2.3), to obtain

$$
W_{t}^{j}+W^{j} W_{z}^{j}=-\frac{P^{j} \rho^{\mathbf{i}}}{\rho^{j}} .
$$

As can easily be deduced, (3.1) implies that $\Delta_{r} W_{\mathrm{c}}^{\circ} / W_{\mathrm{c}}^{0} \sim(B-1)^{2} / 4 A^{2} \ll 1 ; W^{0}$ may then be considered to be independent of the radial coordinate. An analogous condition for the inner flow is $\left(\Delta_{r} W_{\mathrm{c}}^{\mathrm{i}} / W_{\mathrm{c}}^{\mathrm{i}}\right) / 4 \Lambda^{2} \ll 1$. A relationship between $U^{\jmath}$ and $W^{j}$ can then be obtained by radial integration of the continuity equation (2.1):

$$
\begin{aligned}
& U^{\mathrm{i}}(F, z, t)=-\frac{1}{2} F W_{z}^{\mathrm{i}}, \\
& U^{\mathrm{o}}(F, z, t)=\frac{\left(B^{2}-F^{2}\right) W_{z}^{\mathrm{o}}}{2 F} .
\end{aligned}
$$

By substitution of these values in (2.9), equations relating $F$ and $W^{j}$ for the inner and outer liquid, and which do not contain $U^{j}$, are obtained:

$$
\begin{gathered}
\left(F^{2}\right)_{t}+\left(F^{2} W^{\mathrm{d}}\right)_{z}=0, \\
\left(F^{2}\right)_{t}+\left[\left(F^{2}-B^{2}\right) W^{0}\right]_{z}=0 .
\end{gathered}
$$

From these equations a relationship between $W^{i}$ and $W^{0}$ is deduced:

$$
W^{\mathrm{o}}=\frac{F^{2} W^{\mathrm{i}}}{F^{2}-B^{2}},
$$

which is obvious from mass-conservation considerations in a cross-section. This expression allows us to reduce the problem to two equations with two unknowns $F$ and $W^{\mathfrak{L}}$, but even in this form, the problem is too complex and no analytical solution seems obtainable. However, this formulation simplifies, to a great extent, both the linear analysis and the numerical treatment of the problem. 


\subsection{Linear analysis}

The above formulation can be simplified if small velocities and deformations of the liquid bridge are assumed, allowing us to linearize the equations. Let $\epsilon$ be a small parameter, measuring the initial deviation of the interface from the cylinder. Assuming the time dependence to be of the form $\mathrm{e}^{\Omega t}$, the variables of the problem can be expressed as

$$
\left.\begin{array}{c}
W^{j}=\epsilon \mathrm{e}^{\Omega t} w^{j}, \quad F=1+\epsilon \mathrm{e}^{\Omega t} f, \\
P^{o}=\epsilon \mathrm{e}^{\Omega t} p^{o}, \quad P^{\mathrm{i}}=1+\epsilon \mathrm{e}^{\Omega t} p^{\mathrm{i}}
\end{array}\right\}
$$

The equations of motion (3.4), (3.7) and the conditions (2.4), (2.8), (2.10) and (3.9) can be written as

$$
\begin{gathered}
\Omega w^{j}=-\frac{p_{z} \rho^{\mathrm{i}}}{\rho^{j}}, \\
f_{z z}+f=p^{\circ}-p^{\mathbf{i}}, \\
2 \Omega f+w_{z}^{\mathrm{i}}=0 \\
w^{\mathrm{o}}=\frac{w^{\mathrm{i}}}{1-B^{2}} \\
w^{j}( \pm \Lambda)=0, \quad f( \pm \Lambda)=0 .
\end{gathered}
$$

The homogeneous system (3.11)-(3.15) of five equations and five unknowns can be reduced to an equation with one unknown such as

$$
w_{z z z z}^{\mathrm{i}}+w_{z z}^{\mathrm{i}}+2 \Omega_{0}^{2} w^{\mathrm{i}}=0
$$

where

$$
\Omega_{0}^{2}=\Omega^{2}\left(1+\frac{\rho}{B^{2}-1}\right)
$$

and $\rho=\rho^{\circ} / \rho^{\mathrm{i}}$. In this expression $\Omega_{0}$ is the corresponding value of $\Omega$ when there is no bath $(\rho=0)$. The second boundary condition of (3.15) transformed with the aid of (3.13) yields

$$
w^{\mathrm{i}}( \pm \Lambda)=w_{z}^{\mathrm{i}}( \pm \Lambda)=0 .
$$

The problem defined by (3.16) and (3.18) is formally the same as that solved by Meseguer (1983a) for the non-surrounded liquid bridge. It is an eigenvalue problem having non-trivial solutions only for some values of $\Omega_{0}$ related to the parameter $A$ through boundary conditions. Once $\Omega_{0}$, a function only of $A$, is calculated, the time exponent $\Omega$ can be deduced from (3.17) for each value of the parameters $\rho$ and $B$. The non-surrounded-bridge problem has only two possible kinds of solutions: oscillatory movements if $\Omega_{0}$ is an imaginary number, breaking process if it is a real number, and there are no mixed motions in agreement with the inviscid character of the model. These characteristics still remain in the surrounded-liquid-bridge problem, since both $\rho$ and $B$ have real values.

In expressions giving interface shape and axial-velocity dependence on the $z$-and $t$-coordinates (Meseguer $1983 a$ ), bath parameters appear only in the exponent of the time-dependent factor of the solution, $\Omega$. Thus, in the linear approximation, the influence of the bath is limited to a timescale change, defined by (3.17), and no differences in interface shape appear between surrounded and non-surrounded bridges. 
A simple expression of bath influence in breaking process can be deduced by comparing the linear breaking time

$$
T_{\mathrm{lb}}=\frac{1}{\gamma} \ln \epsilon f_{\mathrm{min}}
$$

( $f_{\text {min }}$ stands for the minimum value of $f$, and $\gamma$ is the growth factor) with the linear breaking time of a non-surrounded liquid bridge, $T_{1 \text { bo }}$, which yields

$$
\frac{T_{\mathrm{lb}}}{T_{\mathrm{lb0}}}=\frac{\gamma_{0}}{\gamma}=\left[1+\frac{\rho}{B^{2}-1}\right]^{\frac{1}{2}} \text {. }
$$

This result summarizes the action of the bath. The larger the density of the outer fluid or the closer the position of the wall, the longer the breaking time will be. This phenomenon could be explained in terms of energy interchange between the interface and the fluids. The energy supplied by deformation of the interface, proportional to $\Omega_{0}^{2}$, is transferred to kinetic energy in the inner liquid only, if no bath exists. If the liquid bridge is surrounded, the same deformation energy should be shared by both the inner liquid, whose kinetic energy is proportional to $\Omega^{2}$, and by the bath whose kinetic energy is proportional to $\Omega^{2} \rho /\left(B^{2}-1\right)$, since its velocity is proportional to $\Omega /\left(B^{2}-1\right)$, its volume to $B^{2}-1$ and its density to $\rho$. This interfacial energy sharing explains why the movement of oscillation, as well as breaking, is slowed down. The behaviour predicted by this and other linear models will be compared in $\S 4$.

\subsection{Nonlinear numerical analysis}

The general one-dimensional equations of motion for the liquid bridge and the bath ((2.8), (3.4), (3.7) and 3.9)) have been integrated numerically by using the LaxWendroff method (Roache 1972; Mitchell \& Griffiths 1980). This finite-difference method has been already employed in the case of a non-surrounded liquid bridge (Meseguer 1983a; Meseguer et al. 1983; Meseguer \& Sanz 1985). Before introducing numerical schemes, some manipulation of the general equations is needed in order to simplify the formulation of the problem. Thus the pressure jump can be eliminated by substituting the expressions for $P^{\circ}$ and $P^{1}$, obtained from (3.4), in (2.8), which is transformed into

$$
-\left(W_{t}^{1}+W^{1} W_{z}^{1}\right)+\rho\left(W_{t}^{o}+W^{o} W_{z}^{o}\right)=P_{z}
$$

where $P$ is the capillary pressure, defined by the right-hand side of (2.8). Further simplification $i$, obtained by using new variables defined as $S=F^{2}$ and $Q=F^{2} W^{i}$, proportional respectively to the cross-sectional area and the axial momentum of a slice of the liquid bridge. The outer axial velocity, from (3.9), is $W^{\circ}=Q /\left(S-B^{2}\right)$, and the equations of motion become

$$
\begin{gathered}
S_{t}+Q_{z}=0 \\
S^{-1}\left[Q_{t}+\left(\frac{Q^{2}}{S}\right)_{z}\right]-\rho\left[\frac{Q}{S-B^{2}}\right]_{t}-\frac{1}{2} \rho\left[\left(\frac{Q}{S-B^{2}}\right)^{2}\right]_{z}+P_{z}=0,
\end{gathered}
$$

where the capillary pressure

$$
P=4\left(2 S+S_{z}^{2}-S S_{z z}\right)\left(4 S+S_{z}^{2}\right)^{-\frac{3}{2}}
$$

In (3.23) the underlined terms take into account the existence of the bath. Boundary conditions are

$$
S( \pm \Lambda, t)=1, \quad Q( \pm \Lambda, t)=0 .
$$


Initial conditions state that the liquid bridge, at rest, is slightly disturbed by imposing an antisymmetric deformation to the interface:

$$
S(z, 0)=1+\epsilon(\epsilon-2) \sin \frac{\pi z}{\Lambda}, \quad Q(z, 0)=0 .
$$

The initial shape is assumed to be sinusoidal in the cross-sectional area $S$, so that the volume is conserved; with this choice $\epsilon$ gives the measure of the deformation (in the radius) of the neck of the liquid bridge.

Equation (3.23) may be expressed in a more suitable way by

$$
Q_{t}=Q_{t}^{*}-\mathscr{R}\left(Q_{t}^{*}-\mathscr{L}_{z}\right),
$$

where

$$
\begin{aligned}
& \mathscr{R}=\frac{\rho S}{B^{2}-S(1-\rho)}, \\
& \mathscr{L}=\frac{Q^{2}}{B^{2}-S},
\end{aligned}
$$

and

$$
Q_{t}^{*} \equiv-\left(\frac{Q^{2}}{S}\right)_{z}-S P_{z}
$$

If $\rho=0$ the non-surrounded problem is recovered. The problem to be solved is reduced to (3.22) and (3.27) with conditions $(3.25)$ and (3.26). The numerical scheme already developed for non-surrounded bridges (Meseguer 1983a) still holds, although the axial-momentum equation is transformed because some terms, taking into account the bath influence, are added. Owing to the resemblance between the numerical problems involved in the computations of the dynamic of liquid zones in both cases (with and without bath), a wide use of the methods already developed in the latter has been made.

The implemented program has been run for selected values of the slenderness $A$, the initial disturbance $\epsilon$, and the bath parameters: density ratio $\rho$ and wall position $B$. The large number of parameters being considered generates a large amount of possible configurations. Thus the study has been limited to significant values of $\rho$ and $B$, and also only to the most interesting phenomenon - the breaking process. It should be pointed out that stability limits are not influenced by the outer liquid since they are a static property.

In table 1 some results for the configurations solved are shown. These are the main data from a global point of view : the breaking time $T_{\mathrm{b}}$ (time from start till the neck rupture) and the partial breaking volume $V_{p}$ (volume of liquid placed between the neck and one of the disks to total volume ratio at the breaking time). These (especially the latter) have the virtue of being more easily checked experimentally than other characteristics also obtained from the slice model - for instance the neck evolution versus time or the velocity field.

The results are interesting in that they show clearly the influence of the bath parameters, which can be seen by comparing the results obtained in every case with the corresponding result for the same non-surrounded bridge under an identical initial perturbation. So, the variation of breaking time $T_{\mathrm{b}}$ of a surrounded liquid bridge compared with the value $T_{\mathrm{b} 0}$ for an isolated one versus the position $B$ of the wall is shown in figure 2, together with the linear prediction. As $B$ increases, the breaking time $T_{b}$ approaches that of a non-surrounded bridge, $T_{\mathrm{bo}}$, and they are equal when $B \rightarrow \infty$, that is, no influence of the bath occurs in this limit, which is obviously 


\begin{tabular}{|c|c|c|c|c|c|c|c|c|c|c|c|}
\hline \multirow[b]{2}{*}{$B$} & \multirow[b]{2}{*}{$\rho$} & \multicolumn{2}{|c|}{$A=3.07}$, & \multicolumn{2}{|c|}{$A=3.14, \epsilon=0.2$} & \multicolumn{2}{|c|}{$\Lambda=3.2, \epsilon=0.2$} & \multicolumn{2}{|c|}{$A=3.1, \epsilon=0.3$} & \multicolumn{2}{|c|}{$A=3.1, \epsilon=0.4$} \\
\hline & & $T_{\mathrm{b}}$ & $V_{p}$ & $T_{\mathrm{b}}$ & $V_{p}$ & $T_{\mathrm{b}}$ & $V_{p}$ & $T_{\mathrm{b}}$ & $V_{p}$ & $T_{\mathrm{b}}$ & $V_{p}$ \\
\hline - & 0 & 21.98 & 0.8554 & 13.62 & 0.8491 & 11.52 & 0.8442 & 8.40 & 0.8539 & 4.84 & 0.8566 \\
\hline 1.4 & 1 & 30.69 & 0.8696 & 18.74 & 0.8663 & - & - & - & - & 5.99 & 0.8702 \\
\hline 1.5 & 1 & 28.73 & 0.8661 & 17.60 & 0.8621 & 14.75 & 0.8574 & - & - & 5.79 & 0.8678 \\
\hline 2.5 & 1 & 23.70 & 0.8580 & 14.65 & 0.8522 & - & - & - & - & 5.12 & 0.8596 \\
\hline 2 & 1 & 24.92 & 0.8559 & 15.37 & 0.8546 & 12.96 & 0.8498 & 9.34 & 0.8588 & 5.31 & 0.8617 \\
\hline 2 & 3 & 29.95 & 0.8564 & 18.35 & 0.8611 & - & - & - & - & 6.07 & 0.8674 \\
\hline 2 & 6 & 36.13 & 0.8700 & 22.04 & 0.8666 & - & - & - & - & 7.02 & 0.8718 \\
\hline 2 & 0.5 & 23.50 & 0.8580 & 14.53 & 0.8521 & - & - & - & - & 5.08 & 0.8595 \\
\hline SB & & & & & & & & & & & \\
\hline
\end{tabular}

TABLE 1. Slenderness $A$, initial perturbation amplitude $\epsilon$, wall position $B$, density ratio $\rho$, breaking time $T_{\mathrm{b}}$, partial breaking volume $V_{\mathrm{p}}$, and symbol (SB) indicating the values of $A$ and $\epsilon$ in figures $2-5$.

unrealistic but in agreement with the character of the one-dimensional model, since according to this model both the axial velocity and the kinetic energy of the bath decrease as $1 /\left(B^{2}-1\right)$. However, it should be remembered that the applicability of the model is restricted by the condition $(B-1)^{2} / 4 \Lambda^{2} \ll 1$, the abovementioned limit $B \rightarrow \infty$ being out of the range of applicability.

On the other hand, the lowest values of wall position $B$ are limited by geometrical constraints, at least by the radius of drops produced after the bridge-breaking if the inner liquid is not allowed to touch the lateral wall. The limit imposed by the drop radius is not reached during the breaking process because computing is stopped close to but before splitting occurs (Meseguer \& Sanz 1985). Actually, in the computed breaking process, the final status of the liquid bridge is out of equilibrium (since it corresponds to a dynamic process), and the maximum interface radius in this situation $(\sim 1.4 R)$ is smaller than that of the largest drop produced at the breaking $(\sim 1.5 R)$. Therefore, in the cases solved numerically $B=1.4$ was taken as the lower limit, small enough to show clearly the effect of wall position, although perhaps too small for experimental purposes. Global behaviour of numerical results follows qualitatively the tendency predicted by the linear analysis.

The influence of density ratio is shown in figure 2. In the limiting case $\rho \rightarrow 0$ non-surrounded model values are reached, but the larger the value of $\rho$ the longer the breaking time, owing to growth of outer fluid inertia. As there is no theoretical limit imposed by the one-dimensional model for values of $\rho$, a wide range is taken into account for the sake of completion.

Results showing the influence of bath parameters on partial breaking volumes are plotted in figure 3 . If we consider that in some way the breaking volume is an integral evaluation of the final interface deformation, results demonstrate the small influence of the bath in the interface shape, below $1 \%$ within the range of experimental feasibility $\left(B>2\right.$ is a guess) and model applicability $(B-1)^{2} / 4 A^{2} \ll 1$. This value is of the same order as the errors in most of the experiments reported. As $B$ becomes smaller, the bath influence increases. We should remember that outer and inner axial speeds are related through a quotient involving the distance from the interface to the wall (3.9), so if this distance becomes very small, strong velocities and associated pressure gradients are generated in the outer fluid flow. 

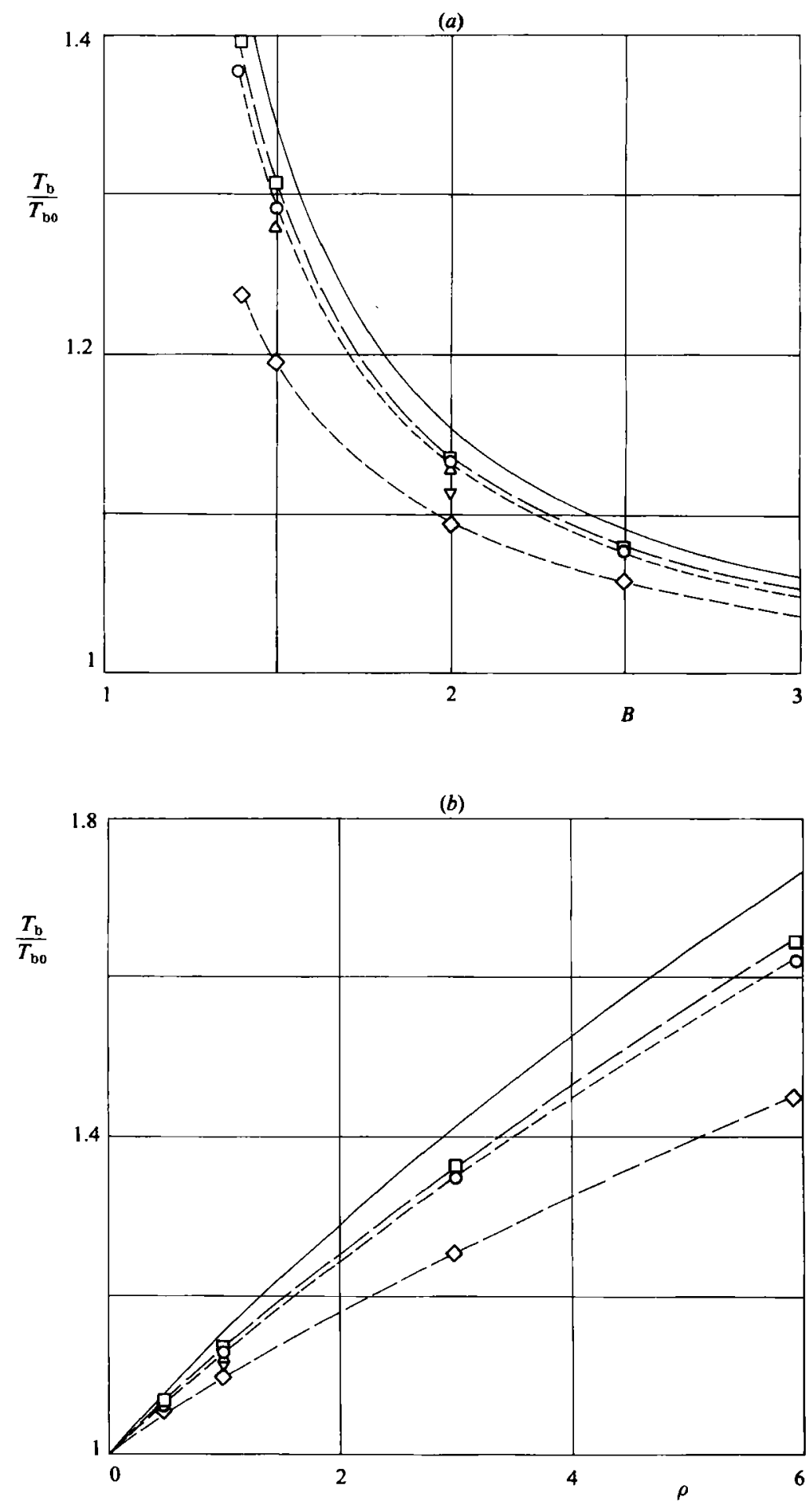

FIGURe 2. Variation of the breaking time $T_{\mathrm{b}}$ of a surrounded liquid bridge, $(a)$ with the wall position $B$ and $(b)$ with the density ratio $\rho$, compared with that of a non-surrounded bridge $T_{\mathrm{b} 0}$ of the same slenderness $A$ and initial perturbation $\epsilon$. $(a) \rho=1 ;(b) B=2$. Solid line, one-dimensional linear model; dashed lines, numerical results from nonlinear one-dimensional model; symbols are defined in table 1 . 
(a)

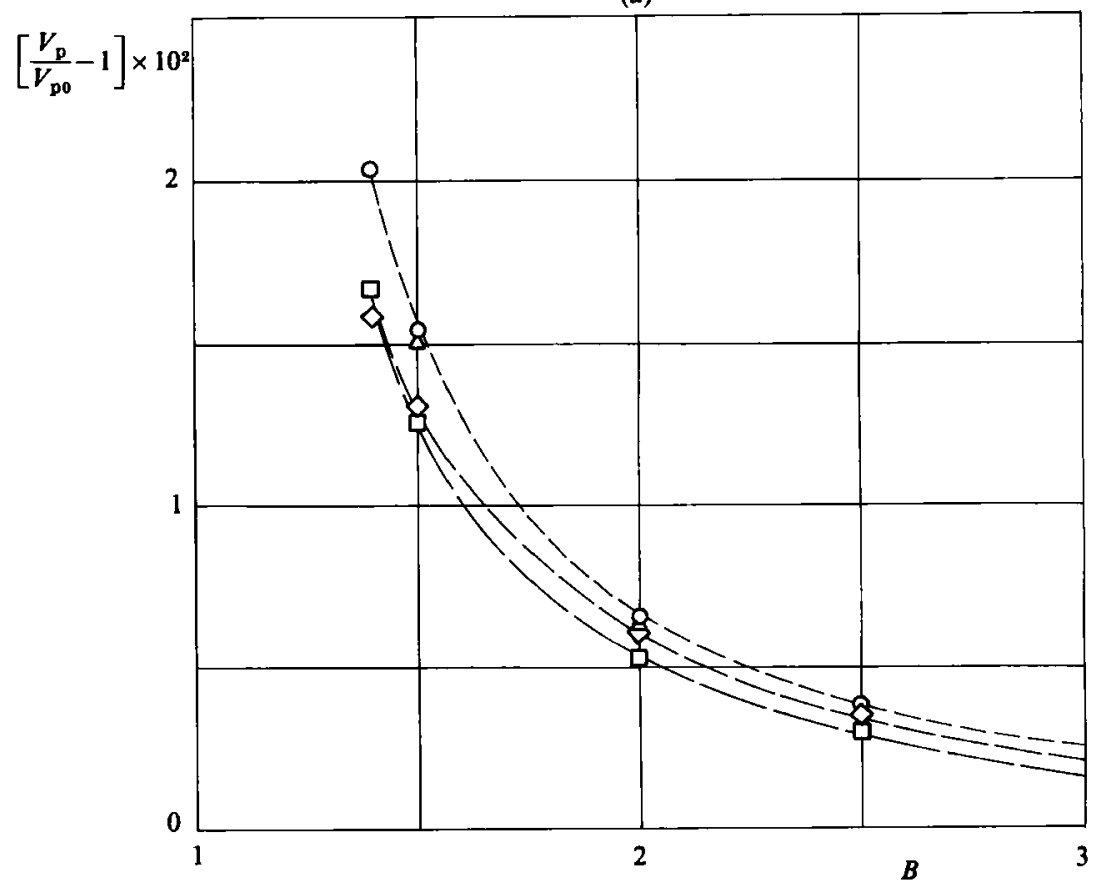

(b)

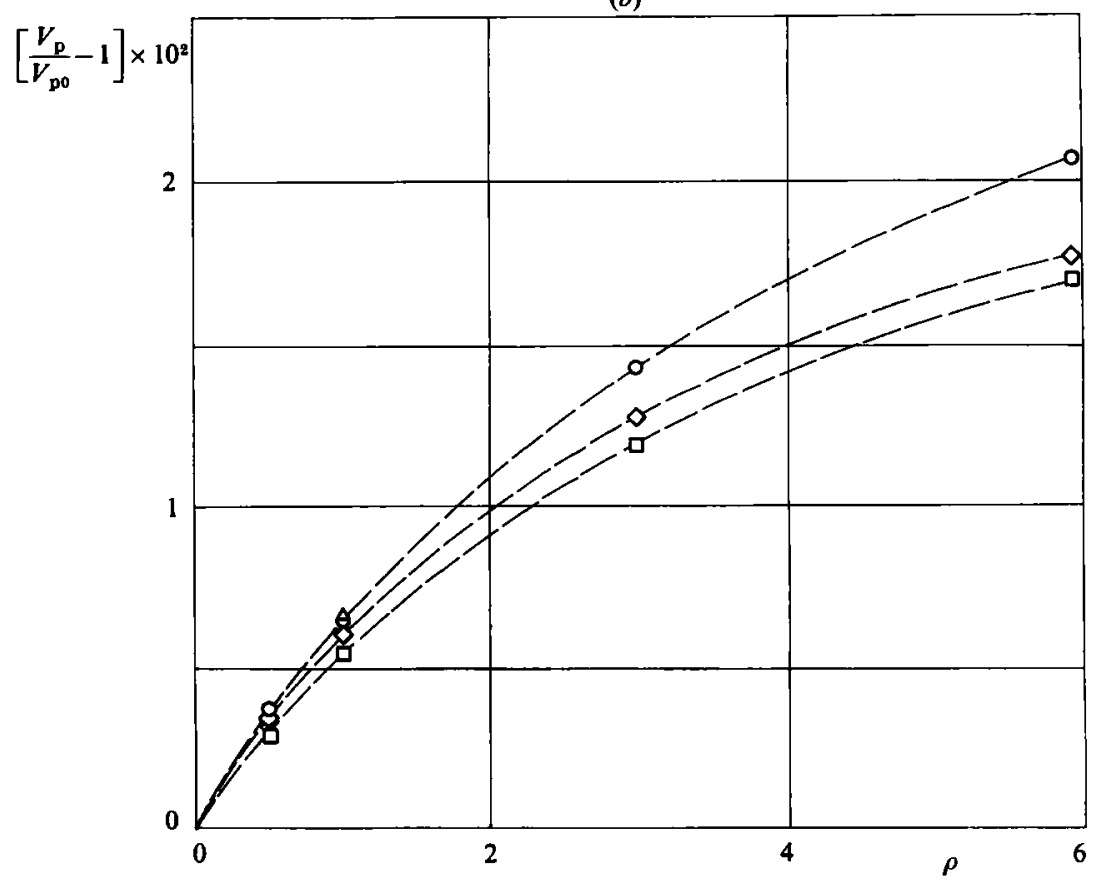

Frgure 3. Variation of the partial breaking volume $V_{\mathrm{p}}$ of a surrounded liquid bridge, $(a)$ with the wall position $B$ and $(b)$ with the density ratio $\rho$, compared with that of a non-surrounded bridge $V_{\mathrm{po}}$ of the same slenderness $A$ and initial perturbation $\epsilon$. (a) $\rho=1 ;(b) B=2$. Symbols are defined in table 1. 


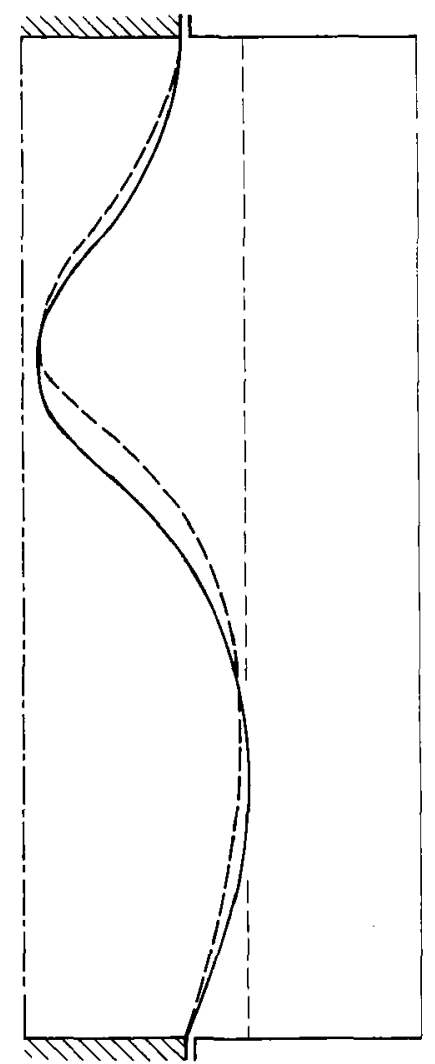

Fioure 4. Interface shapes, close to the breaking, of two liquid bridges of the same slenderness $(\Lambda=3.14)$, starting with the same initial perturbation $(\epsilon=0.2)$, surrounded by a density-matched liquid $(\rho=1)$. The lateral wall is placed at $B=1.4$ (dashed line) and $B=2.5$ (solid line) respectively.

The effect of the wall position on the interface shape is seen in figure 4 , which shows the deformation of two liquid bridges of the same $A$ and equal initial perturbation $\epsilon$, but surrounded by a bath whose lateral wall is placed at $B=1.4$ and $B=2.5$ respectively. In the first case the effect of the wall can be seen quite clearly, the interface keeps close to it but a small gap exists, through which the outer liquid moves from the largest section towards the neighbourhood of the neck section. On the other hand, the wall avoids growing to the largest cross-section, which causes the neck to move towards the nearest disk, and thence partial breaking volumes increase. In the second case $(B=2.5)$ the interface shape is nearly the same as that of the non-surrounded liquid bridge, as reflected by the difference in the breaking volume, some $0.3 \%$.

To enhance the appreciation of the influence of the $A$-and $\epsilon$-parameters, let us make some further considerations. As can be seen from the previous results, the linear theory and numerical results show a similar behaviour. This fact suggests the use of the relationship predicted by the linear analysis to reduce the numerical results. Let us denote the quantity $\left[\left(T_{\mathrm{b}} / T_{\mathrm{b} 0}\right)^{2}-1\right]\left(B^{2}-1\right) / \rho$ as the reduced breaking time $T_{\mathrm{r}}$ (in linear theory $\left.T_{\mathrm{r}}=1\right)$. If the reduced breaking time is plotted versus the parameter $\rho /\left(B^{2}-1\right)$, as shown in figure 5, within each group of results, identified by the same $A$ and $\epsilon$, two sets can be distinguished, depending on whether $\rho$ or $B$ varies. Their 


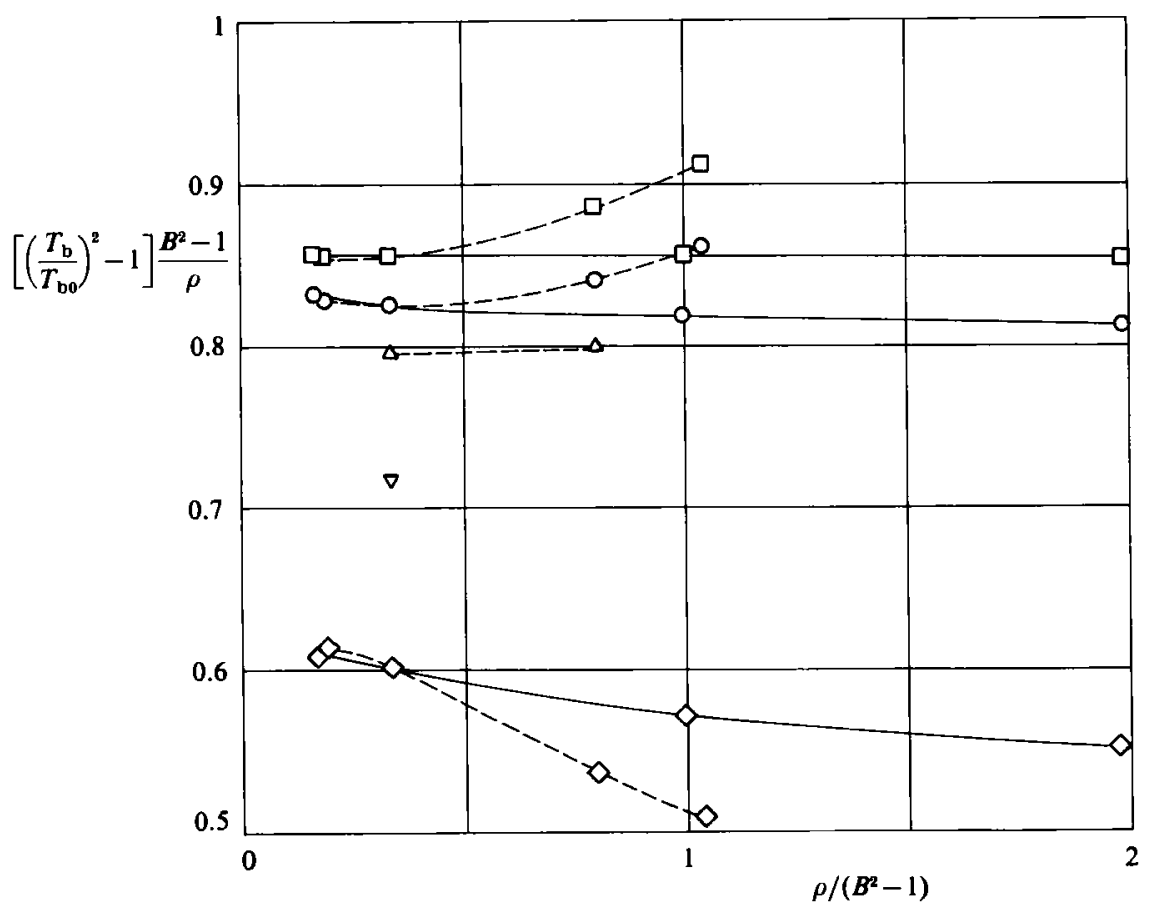

FigURE 5. Reduced breaking time $\left[\left(T_{\mathrm{b}} / T_{\mathrm{b} 0}\right)^{2}-1\right]\left(B^{2}-1\right) / \rho$ versus the parameter $\rho /\left(B^{2}-1\right)$. Symbols are defined in table 1. Solid (dashed) lines join points with the same density ratio $\rho=1$ (same position of the wall, $B=2$ ).

influence is different because of the roles they play: $\rho$ is a parameter affecting the whole bath, whereas $B$ induces a distributed effect, depending on the distance between the interface and the wall. In addition to the influence of $\rho$ and $B$, effects produced by $\Lambda$ and $\epsilon$ are also clearly shown. As could be foreseen from the behaviour in the non-surrounded case, as $A$ and $\epsilon$ increase, the reduced breaking time decreases; that is, the more unstable the initial configuration, the shorter the reduced breaking time.

\subsection{Nonlinear asymptotic analysis}

As deduced from the linear analysis, the first stability change, $\Omega=0$, occurs at the same point as that in the non-surrounded case, $A=\pi$. The character of the bifurcation to unstable shapes and the dynamics in the neighbourhood of this point have been extensively studied, both numerically (Meseguer 1983 $a$ ) and asymptotically (Rivas \& Meseguer 1985) in the case of non-surrounded bridges. Following the same method as in the latter paper, it can be shown that the existence of an outer fluid does not modify the curve $\epsilon(\Lambda)$ of unstable equilibrium shapes in the $(\Lambda, \epsilon)$-diagram, and affects only the timescale of the motion in the same way as predicted by linear analysis (3.17). The idea is that unstable equilibrium shapes are determined by the static problem, that is, by the solutions of (3.24) with constant $P$. Therefore the outer liquid has no influence in them, and only plays a role in the dynamics. 


\section{Linear three-dimensional model}

The one-dimensional model analysed in $\$ 3$ offers an approach to the study of the dynamics (oscillation motion and breaking process) of liquid bridges, although some conditions of validity should be satisfied, restraining to slender bridges the applicability of both linear and nonlinear approaches derived from this model. Concerning oscillatory motions, from the experimental point of view, slender liquid bridges present specific problems which can be avoided by using medium-length bridges, to which the applicability of the one-dimensional model is not directly drawn. A theoretical model of oscillations without this restriction is the infinite-column model (Bauer 1982), based on the capillary-jet theory. However, it is valid only for non-anchored liquid bridges, thus it does not satisfy simultaneously the two boundary conditions at the disks more usual in experiments (zero normal velocity and anchored interface).

In this section, a three-dimensional linear model, without the abovementioned restrictions, is presented. As this model retains all the significant characteristics of the phenomenon (in the inviscid case), its results should be closer to reality than other models, which contain additional simplifying hypotheses.

\subsection{Linear analysis}

Let us take as starting point the formulation of the surrounded-liquid-bridge problem stated in $\$ 2$. To solve the problem the formulation has been linearized, assuming small interface deformations and velocities, obtaining a free boundary problem for the pressure. As this kind of problem has no standard solution, the procedure to find the possible solutions will be as follows. As a first step, general solutions for both the inner and outer liquids, satisfying the boundary conditions at the solid surfaces and at the axis of the bridge, are built up. In a second step the coefficients appearing in these solutions are calculated by using boundary conditions at the interface.

In the same way as in $\$ 3.2$, let $\epsilon$ be a small parameter measuring the initial deviation of the interface from the cylinder. Assuming the dependence on time as $\mathrm{e}^{\Omega t}$, the variables of the problem can be expressed as follows:

$$
\left.\begin{array}{c}
U^{j}=\epsilon \mathrm{e}^{\Omega t} u^{j}, \quad W^{j}=\epsilon \mathrm{e}^{\Omega t} w^{j}, \\
P^{\mathbf{i}}=1+\epsilon \mathrm{e}^{\Omega t} p^{\mathrm{i}}, \quad P^{\mathrm{o}}=\epsilon \mathrm{e}^{\Omega t} p^{\mathrm{o}}, \\
F=1+\epsilon \mathrm{e}^{\Omega t} f .
\end{array}\right\}
$$

By substitution in the equations of $\$ 2$, the problem reduces to

$$
\begin{gathered}
u_{r}^{j}+\frac{u^{j}}{r}+w_{z}^{j}=0 \\
\Omega u^{j}=-\frac{p_{r}^{j} \rho^{\mathbf{1}}}{\rho^{j}} \\
\Omega w^{j}=-\frac{p_{z}^{j} \rho^{\mathrm{i}}}{\rho^{j}} .
\end{gathered}
$$

Boundary conditions at the limiting surfaces are

$$
w^{j}(r, \pm A)=0, \quad u^{o}(B, z)=0
$$


and at the axis of the bridge

$$
u^{i}(0, z)=0, \quad w_{r}^{i}(0, z)=0 .
$$

At the linearized interface position $r=1$ boundary conditions are that the pressure jump should balance the capillary pressure

$$
f_{z z}+f=-p^{1}(1, z)+p^{\circ}(1, z),
$$

and kinematic compatibility,

$$
\Omega f-u^{j}(1, z)=0 .
$$

Conditions for the interface itself are volume preservation;

$$
\int_{-A}^{A} f \mathrm{~d} z=0
$$

and anchored triple contact line,

$$
f( \pm \Lambda)=0 \text {. }
$$

By substituting (4.3) and (4.4) in the continuity equation (4.2) and in (4.5) and (4.6), the problem is reduced to

$$
p_{r r}^{\jmath}+\frac{p_{r}^{\jmath}}{r}+p_{z z}^{\jmath}=0
$$

with the boundary conditions

$$
p_{z}^{\jmath}(r, \pm \Lambda)=p_{r}^{\mathrm{i}}(0, z)=p_{r}^{\mathrm{o}}(B, z)=0 .
$$

The more general solution of (4.11) for the inner and the outer problem which satisfies (4.12) is

$$
\begin{aligned}
& p^{\mathbf{i}}=g^{\mathrm{i}}+\sum_{n=1}^{\infty} \Omega^{2} a_{n} I_{0}\left(l_{n} r\right) \cos l_{n}(z+A), \\
& p^{\mathrm{o}}=g^{\mathrm{o}}+\sum_{n=1}^{\infty} \Omega^{2} b_{n}\left[K_{0}\left(l_{n} r\right)+I_{0}\left(l_{n} r\right) \frac{K_{1}\left(l_{n} B\right)}{I_{1}\left(l_{n} B\right)}\right] \cos l_{n}(z+A),
\end{aligned}
$$

where $l_{n}=n \pi / 2 A ; g^{1}, g^{\circ}, a_{n}, b_{n}$ are arbitrary constants, and $I_{0}(x), I_{1}(x), K_{0}(x), K_{1}(x)$ are the modified Bessel functions of zero and first order (indicated by the subscript) and first and second kind. The expressions (4.13) and (4.14) satisfy the boundary conditions at the solid surfaces and at the axis of symmetry. Should they be the solutions of the problem, they must also satisfy the conditions at the interface, through which the arbitrary constants involved in the solution can be determined. Let us suppose that $f$ is developed in a cosine series, like $p^{j}$. Then $3 n+3$ constants appear, $n$ from $f, 2 n+1$ in the pressure jump $\left(a_{n}, b_{n}, g^{1}-g^{0}\right)$, and two in integrating (4.7). To determine them there are three conditions $((4.9)$ and $(4.10))$, and three identities ((4.7) and (4.8)), which can be converted by orthogonality properties to $3 n$ algebraic equations. In this way an algebraic homogeneous system is built up, with the same number of equations and unknowns, it will then have solutions only for values of the parameters involved ( $\Omega$ and $\Lambda$ ) that satisfy some secular equation.

Although in a general case it would be complicated to find the solutions of this problem, the algebraic manipulation involved in that case can be reduced somehow. Thus the velocities $u^{j}$ can be eliminated from (4.8) by using (4.3), obtaining

$$
\Omega^{2} f=-p_{r}^{1}(1, z)=-\frac{p_{r}^{o}(1, z)}{\rho} .
$$


From the first equality of (4.15), $f$ should have the form

$$
f=-\sum_{n=1}^{\infty} a_{n} l_{n} I_{1}\left(l_{n}\right) \cos l_{n}(z+\Lambda),
$$

and from the second equality a relationship between $a_{n}$ and $b_{n}$ is obtained:

$$
b_{n}=-a_{n}\left[\frac{K_{1}\left(l_{n}\right)}{I_{1}\left(l_{n}\right)}-\frac{K_{1}\left(l_{n} B\right)}{I_{1}\left(l_{n} B\right)}\right]^{-1}
$$

On the other hand, the condition of pressure balance at the interface can be written with the aid of $(4.13),(4.14)$ and (4.17):

$$
f_{z z}+f=-g_{0}-\sum_{n=1}^{\infty} \Omega^{2} a_{n} I_{0}\left(l_{n}\right) S_{n} \cos l_{n}(z+\Lambda),
$$

where $g_{0}=g^{i}-g^{0}$ and

$$
S_{n}=1+\rho\left[\frac{K_{0}\left(l_{n}\right)}{I_{0}\left(l_{n}\right)}+\frac{K_{1}\left(l_{n} B\right)}{I_{1}\left(l_{n} B\right)}\right]\left[\frac{K_{1}\left(l_{n}\right)}{I_{1}\left(l_{n}\right)}-\frac{K_{1}\left(l_{n} B\right)}{I_{1}\left(l_{n} B\right)}\right]^{-1}
$$

The solution of the ordinary differential equation (4.18) is

$$
f=-g_{0}+a \cos z+b \sin z-\sum_{n=1}^{\infty} \Omega^{2} a_{n} S_{n} I_{0}\left(l_{n}\right)\left(1-l_{n}^{2}\right)^{-1} \cos l_{n}(z+\Lambda) .
$$

At this point the problem is reduced to $n+3$ unknowns $\left(a_{n}, g_{0}, a, b\right)$ plus $n+3$ conditions, $n$ from the identity between (4.16) and (4.20), and three from (4.9) and (4.10). To identify (4.16) and (4.20) the developments of $\cos z$ and $\sin z$ in series of $\cos l_{n}(z+A)$ are needed:

$$
\begin{aligned}
& \sin z=2 \frac{\cos A}{\Lambda} \sum_{m=0}^{\infty}\left(1-l_{2 m+1}^{2}\right)^{-1} \cos l_{2 m+1}(z+\Lambda), \\
& \cos z=\frac{\sin A}{A}\left[1+2 \sum_{m-1}^{\infty}\left(1-l_{2 m}^{2}\right)^{-1} \cos l_{2 m}(z+\Lambda)\right] .
\end{aligned}
$$

Equating the coefficients of $\cos l_{n}(z+A)$ in (4.16) and (4.20) with the aid of (4.21) and (4.22), the unknowns $a_{n}$ can be obtained:

$$
\begin{gathered}
a_{2 m+1}=2 b \frac{\cos \Lambda}{\Lambda}\left[\Omega^{2} j_{2 m+1}-k_{2 m+1}\right]^{-1}, \\
a_{2 m}=2 a \frac{\sin A}{\Lambda}\left[\Omega^{2} j_{2 m}-k_{2 m}\right]^{-1},
\end{gathered}
$$

where

$$
\begin{aligned}
& j_{m}=I_{0}\left(l_{m}\right) S_{m}, \\
& k_{m}=l_{m}\left(1-l_{m}^{2}\right) I_{m}\left(l_{m}\right) .
\end{aligned}
$$

Now only 3 unknowns ( $a, b$ and $g_{0}$ ) and three conditions ((4.9) and (4.10)) remain. They can be written in the following way:

$$
\begin{gathered}
-g_{0}+a \cos A+b \sin A-\sum_{n=1}^{\infty} \Omega^{2} a_{n} S_{n} I_{0}\left(l_{n}\right)\left(1-l_{n}^{2}\right)^{-1} \cos n \pi=0 \\
-g_{0}+a \cos A-b \sin A-\sum_{n=1}^{\infty} \Omega^{2} a_{n} S_{n} I_{0}\left(l_{n}\right)\left(1-l_{n}^{2}\right)^{-1}=0 \\
-g_{0} A+a \sin A=0 .
\end{gathered}
$$


With some manipulation $g_{0}$ can be eliminated, and separated equations are obtained for $a$ or $b$, depending on whether $n$ is odd or even:

$$
\begin{gathered}
b \sin A+\sum_{m=0}^{\infty} \Omega^{2} a_{2 m+1} i_{2 m+1}=0 \\
a \cos A\left(1-\frac{\tan A}{\Lambda}\right)-\sum_{m-1}^{\infty} \Omega^{2} a_{2 m} i_{2 m}=0
\end{gathered}
$$

where

$$
i_{m}=S_{m} I_{0}\left(l_{m}\right)\left(1-l_{m}^{2}\right)^{-1} \text {. }
$$

If in (4.30) and (4.31) the expressions for $a_{n}$ given by (4.23) and (4.24) are substituted, two secular equations can be obtained:

$$
\begin{gathered}
\frac{1}{2} \Lambda \tan \Lambda+\Omega^{2} \sum_{m=0}^{\infty} i_{2 m+1}\left(\Omega^{2} j_{2 m+1}-k_{2 m+1}\right)^{-1}=0 \\
\frac{\Lambda-\tan \Lambda}{2 \tan \Lambda}-\Omega^{2} \sum_{m=1}^{\infty} i_{2 m}\left(\Omega^{2} j_{2 m}-k_{2 m}\right)^{-1}=0
\end{gathered}
$$

Conditions (4.34) or (4.33) hold respectively depending on whether the interface deformation is symmetric ( $n$ even) or antisymmetric with respect to the mean plane between disks.

The time-dependent part of the solution, $\mathrm{e}^{\Omega t}$, gives the character of the evolution. In general, the exponent $\Omega$ is a complex number, $\Omega=\gamma+i \omega$, where both the growth factor $\gamma$ and the pulsation $\omega$ are real numbers; therefore, in principle, compound motions, in which oscillation and growth or decay motions are present together, could arise. However, it is possible to demonstrate that the only values of $\Omega$ that can satisfy (4.33) or (4.34) are either real or imaginary numbers, in such a way that either periodic or growing and decaying solutions are allowed but compound motions are avoided, in agreement with the inviscid character of the model. Let us substitute $\Omega=\gamma+i \omega$ in (4.33) and (4.34), and split them into real and imaginary parts. The latter, which comes from the expression under the summation sign, is similar in both equations, and can be written in the form:

$$
\gamma \omega \sum_{n} l_{n} S_{n} I_{0}\left(l_{n}\right) I_{1}\left(l_{n}\right)\left\{\left[\left(\gamma^{2}-\omega^{2}\right) j_{n}-k_{n}\right]^{2}+\left[2 \gamma \omega j_{n}\right]^{2}\right\}^{-1}=0
$$

As the sum is always a positive quantity, since all its terms are positive, the unique solutions allowed are $\gamma=0$ or/and $\omega=0$, as stated above. In addition to that, as in (4.33) and (4.34), the quantity $\Omega^{2}$ appears, so two suitable values will be obtained, $\Omega= \pm \gamma$ in the case of growing motions, and $\Omega= \pm \mathrm{i} \gamma$ in oscillatory motions. These two solutions can be combined to satisfy initial conditions (zero velocity or zero deformation).

\subsection{Theoretical results}

The variation of $\omega$ with $\Lambda$ calculated by using (4.33) and (4.34), for the case of a non-surrounded bridge, is shown in figure 6 .

The values of $\Lambda$ at which stability changes occur can be obtained by substituting $\Omega=0$ in (4.33) and (4.34) to give

$$
\begin{aligned}
\tan A & =0, \\
\tan A & =A,
\end{aligned}
$$




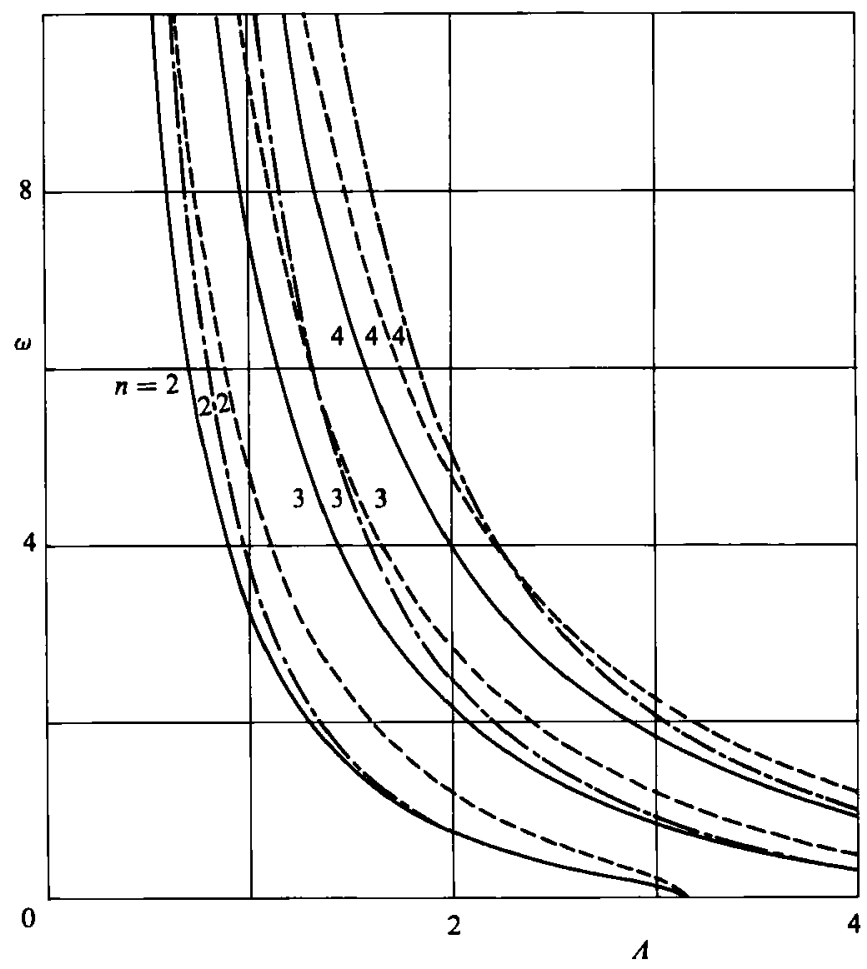

Figure 6. Pulsation $\omega$ versus slenderness, $A$ in the first three oscillation modes of a non-surrounded liquid bridge obtained from several linearized models: three-dimensional (solid line), infinite columns (dashed line), one-dimensional (dot-dashed line). $n$ indicates the number of half-waves of the interface deformation.

results already known in the case of jets and non-surrounded liquid bridges (Rayleigh 1945; Martinez 1978). In the following we will discuss only the oseillatory solutions, although linear models are valid for the initial part of the breaking process as well. The interface deformation can be calculated by substituting the values of $a_{n}$ from (4.23) or (4.24) in (4.16), depending on the kind of deformation being considered, in the form

$$
\begin{aligned}
& f(z)=-2 b \frac{\cos \Lambda}{\Lambda} \sum_{m=0}^{\infty} l_{2 m+1} I_{1}\left(l_{2 m+1}\right)\left[\Omega_{\mathrm{p}}^{2} j_{2 m+1}-k_{2 m+1}\right]^{-1} \cos l_{2 m+1}(z+\Lambda), \\
& f(z)=-2 a \frac{\sin \Lambda}{\Lambda} \sum_{m=1}^{\infty} l_{2 m} I_{1}\left(l_{2 m}\right)\left[\Omega_{\mathrm{p}}^{2} j_{2 m}-k_{2 m}\right]^{-1} \cos l_{2 m}(z+\Lambda),
\end{aligned}
$$

where $\Omega_{\mathrm{p}}$ is one of the values that can be obtained as a solution of the corresponding equation for the value of $\boldsymbol{A}$ considered. With each $\boldsymbol{\Omega}_{\mathrm{p}}$ a different mode of oscillation is associated. The coefficients $a$ and $b$ can be deduced from the amplitude of the initial velocity field or deformation. In figure 7 two typical deformations are plotted.

As indicated above, the time variation is given by the $\mathrm{e}^{\Omega t}$ term, which represents either oscillatory or divergent motions, depending on $\Omega$. A $\frac{1}{2} \pi$ difference in phase between the deformation and the velocity field exists in oscillation, showing the well-known phenomenon of zero deformation when the velocity reaches a maximum and vice versa. Pressure or velocity fields can be determined in a similar way to that followed for deformations. 


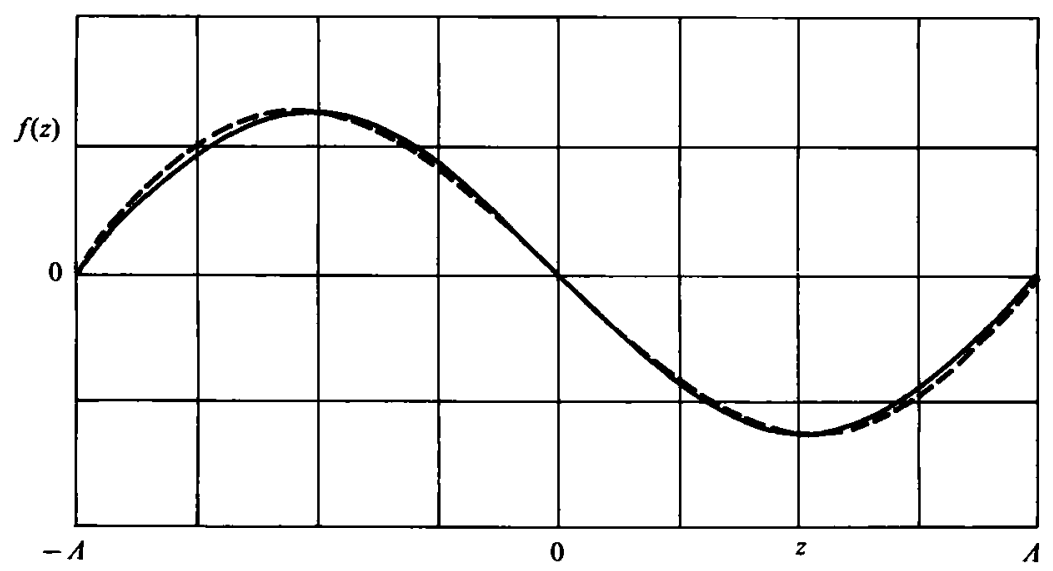

Fioure 7. Interface deformations (from cylindrical shape) of surrounded liquid bridges of slenderness $A=2.9$ (solid line) and $A=1$ (dashed line). First oscillation mode.

(a)

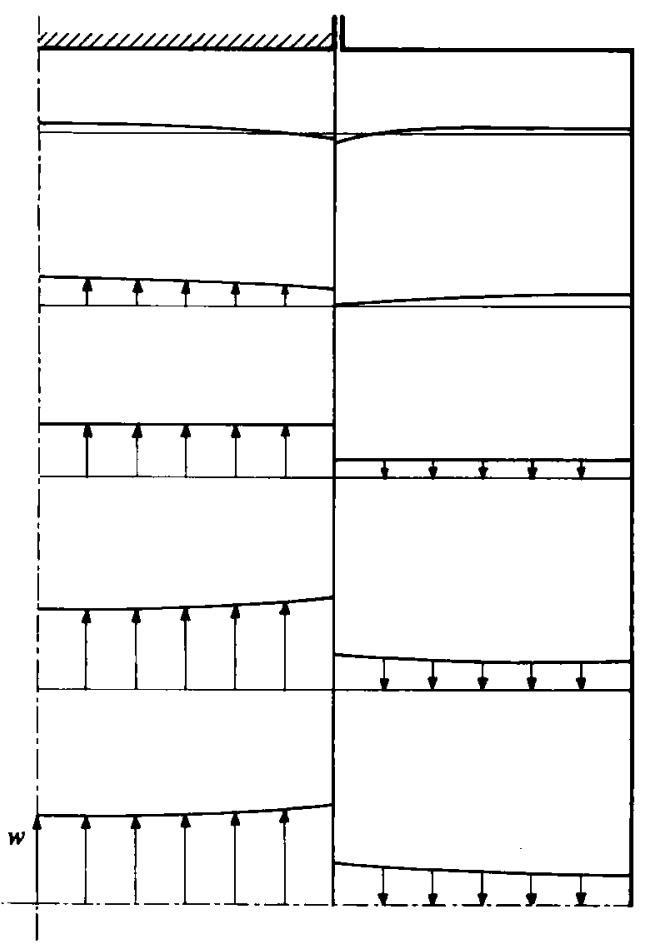

(b)

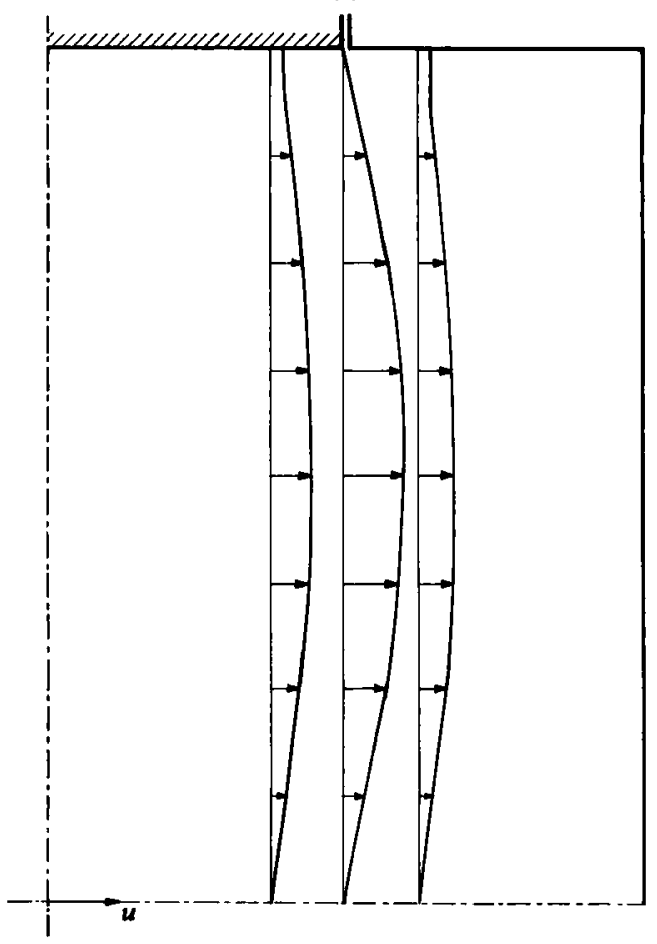

Fiadre 8. (a) Axial-velocity transverse profiles and (b) radial-velocity longitudinal profiles, in a surrounded liquid bridge of slenderness $A=2.9$ and wall position $B=2$. First oscillation mode. Only half of the bridge is presented owing to symmetry.

In figures 8-10 several velocity profiles are plotted for two bridges of different slenderness, one tall and the other short, in oscillation. As can be seen in figure 8, in the former case radial variations of axial velocity are small, except in the neighbourhood of the disks near the interface (in both the inner and outer liquid), in which the axial velocity changes in sign. In the case of a short bridge, as shown in 

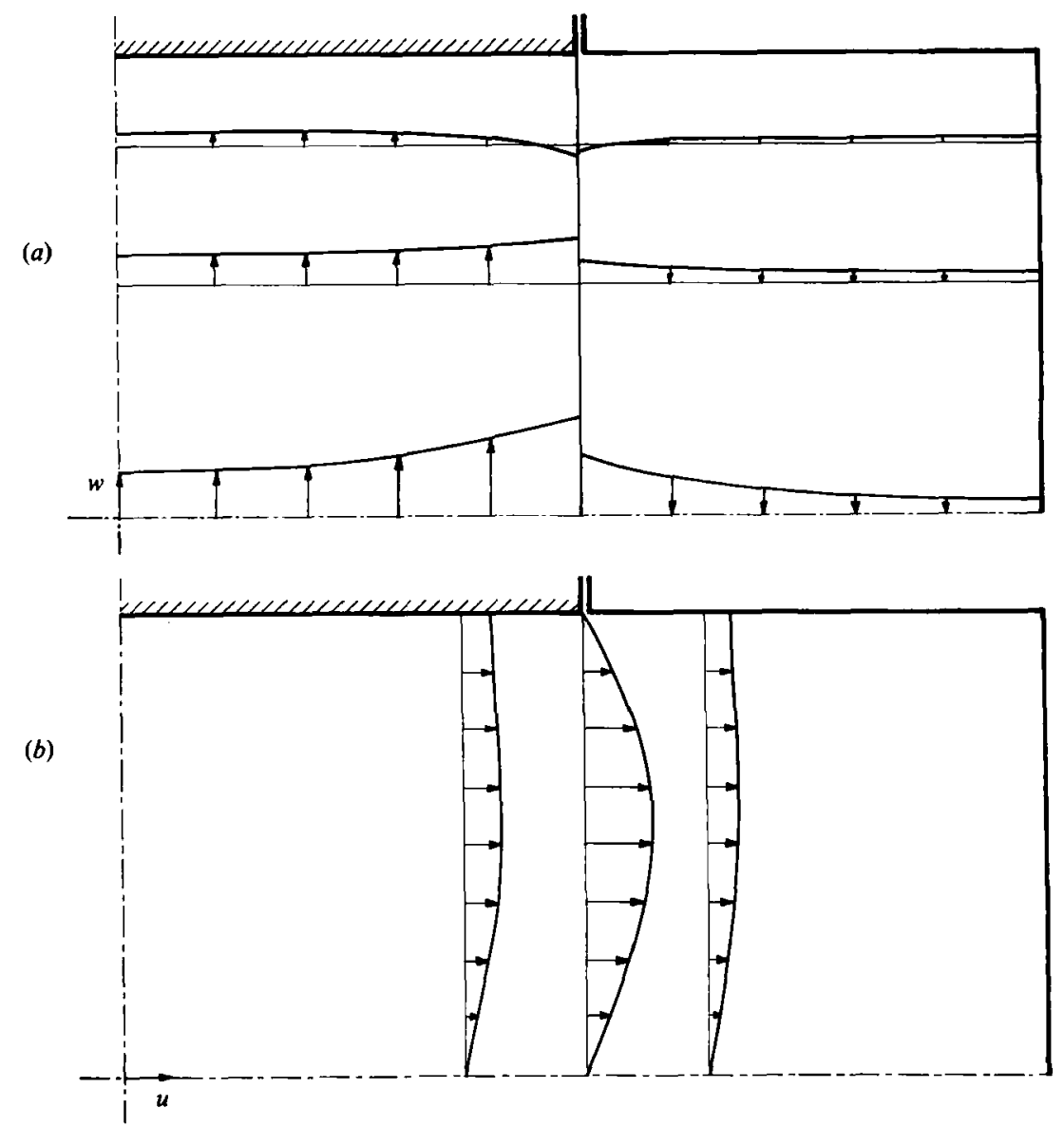

Froure 9. (a) Axial-velocity transverse profiles and $(b)$ radial-velocity longitudinal profiles, in a surrounded liquid bridge of slenderness $A=1$ and wall position $B=2$. First oscillation mode. Only half of the bridge is presented owing to symmetry.

figures 9 and 10, the axial velocity and its radial variations are of the same order of magnitude all along the bridge, and the region in which $w$ changes sign is larger and more important than in the previous case.

Global features of the motion are the differences in sign between $w^{0}$ and $w^{1}$, whereas it remains the same within each medium, except in the aforementioned region near the contact line. In the case of slender bridges, as shown in figure 8, longitudinal profiles of radial velocity are almost similar to the interface deformation, except near the disk, where the velocity has a non-zero value, although small if compared with the maximum value (of course a strict proportionality should exist between velocity profiles at $r=1$ and interface deformations). In contrast, this phenomenon does not happen in short bridges, and clear differences arise between velocity profiles and bridge deformations; for instance, the velocity at the disks has a similar intensity to the maximum velocity, except in a small region near the interface. This fact appears clearly in figure 10. In slender bridges, except near to the disks, radial-velocity transverse profiles are linear, as assumed for the one-dimensional model - a characteristic which is lost in short bridges. 
(a)
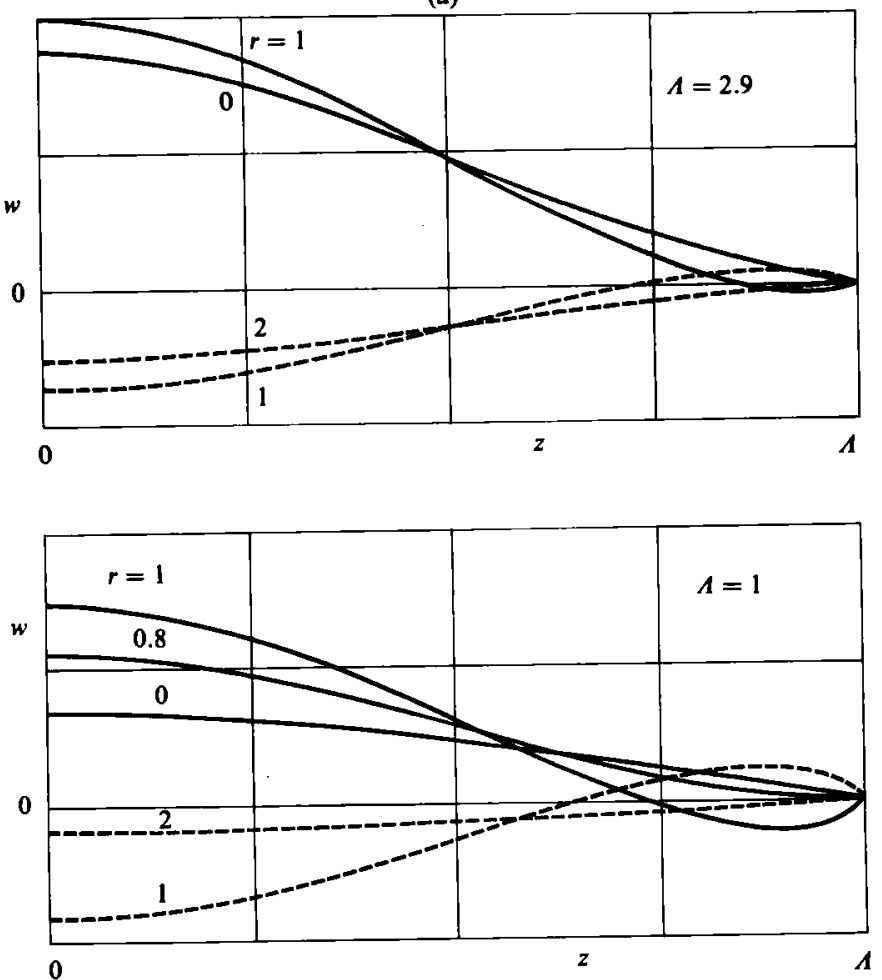

(b)
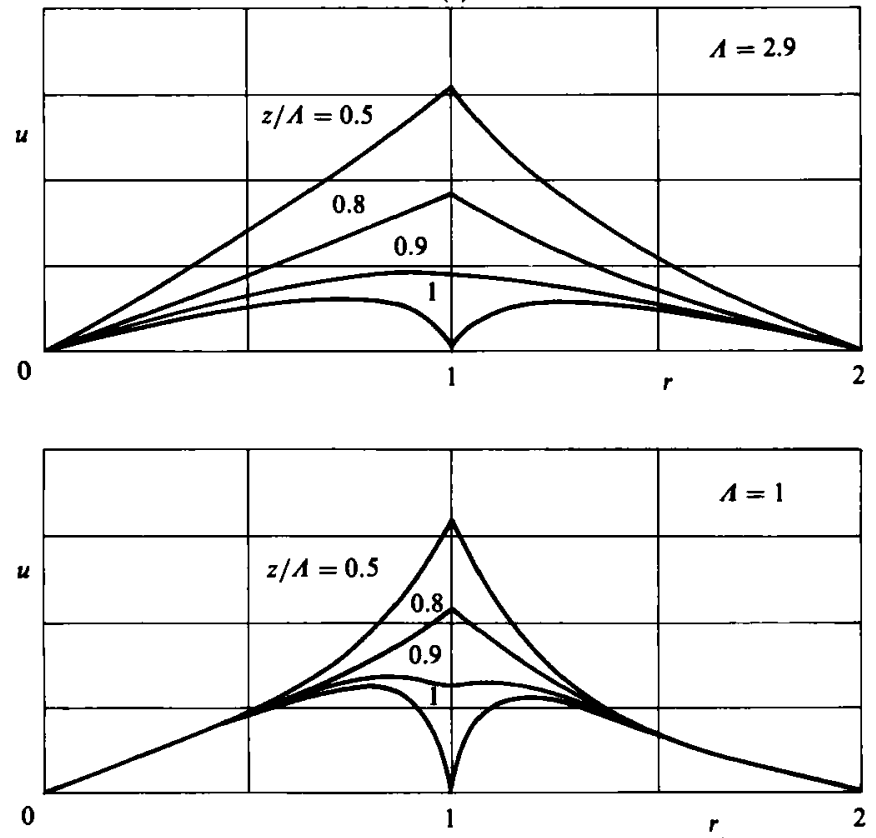

Figure 10. (a) Variation of axial velocity $w$ with $z$-coordinate, for several values of $r$, inside the liquid bridge (solid line) and in the bath (dashed line), only half of the bridge is presented owing to symmetry properties. (b) Variation of radial velocity $u$ with $r$-coordinate, inside the bridge $(r<1)$ and in the bath $(r>1)$, for several values of $z / A$. Wall position at $B=2$. First oscillation mode. 

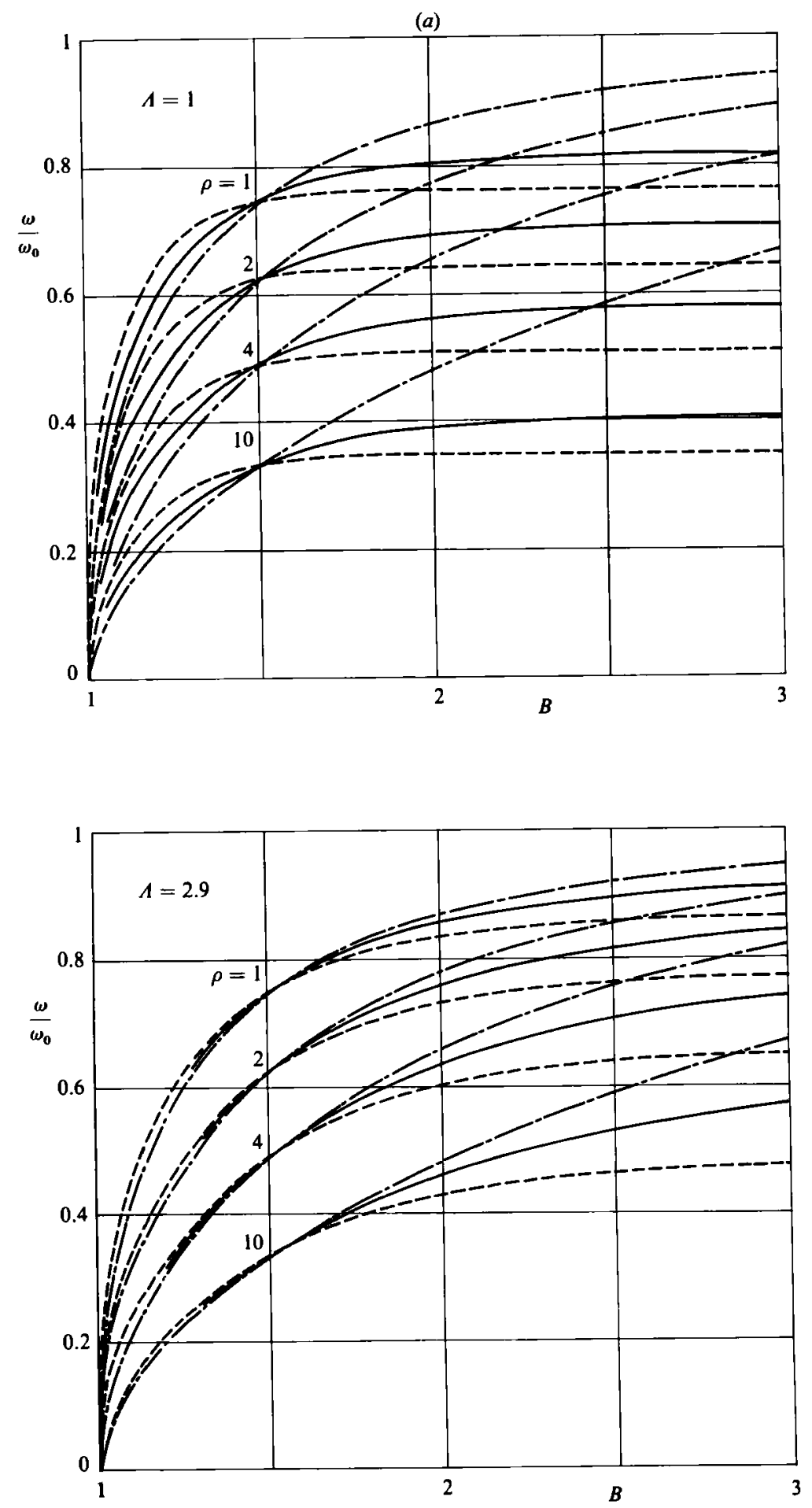

Figure $11(a)$. For description see opposite. 

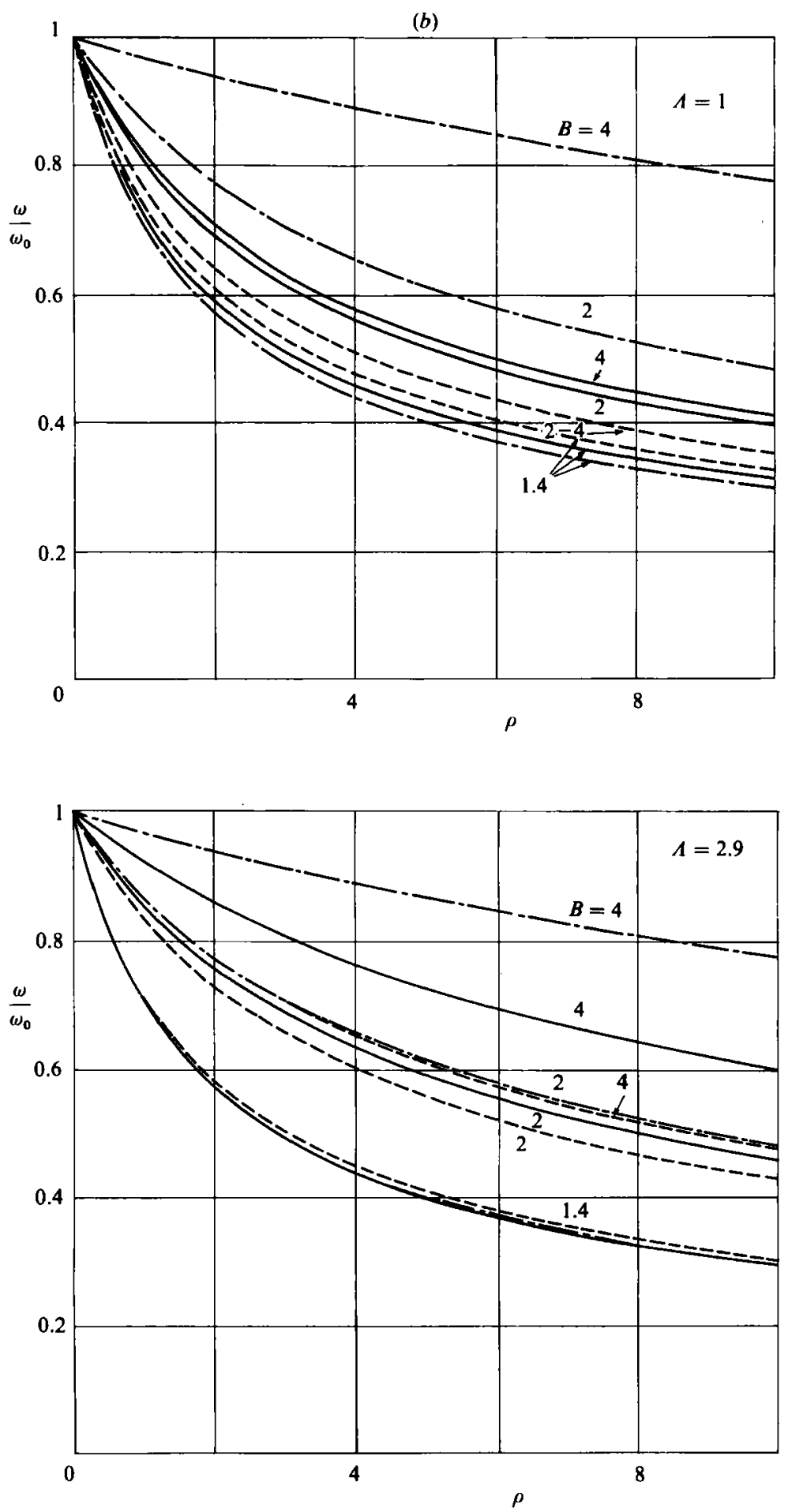

Frgure 11. (a) Pulsation $\omega$ versus wall position $B$ and $(b)$ pulsation versus density ratio $\rho$, compared with non-surrounded liquid-bridge pulsation $\omega_{0}$. First oscillation mode of a surrounded bridge of slenderness $\boldsymbol{A}$. Results obtained from several linearized models: three-dimensional (solid line), infinite columns (dashed line) and one-dimensional (dot-dashed line). 
In a low-viscosity liquid the jump in $w$ across the interface would give rise to a non-steady boundary layer on both sides of the interface, which accommodates the shear stress.

\subsection{Bath influence}

The influence of the outer liquid appears mainly in the time-dependent part of the solution. As shown in figure 11, the resonant frequency $\omega$ decrease as the density ratio $\rho$ increases or the wall position approaches the interface. In contrast, in the space-dependent part of the solution for the interface deformation and the velocity field, shown in figures $7-10$, the results obtained almost coincide in spite of the differences in the density ratio, whose small influence cannot be appreciated in these figures. The variation of $B$ gives rise to a modification of the velocity field within the bath, due to the change in geometry, but it has no influence in the inner velocity field, as shown in figure 12 .

Additional details of bath influence should be pointed out. As shown in figure 11, the variation of $\omega$ with $B$ is quite clear, keeping almost constant for $B>3$ and decaying from that value to zero as $B$ approaches unity. This characteristic is explained by the exponential decay of the terms of $S_{n}$ containing $B$, which is responsible for the fading away of the variation with $B$. It suggests that if walls were far from the bridge (meaning $B \sim 2$ or 3 - the validity limit of the one-dimensional model) the shape of the container would have no influence on the motion.

The qualitative influence of the bath has already been explained in $\$ 3.2$, and the main features remarked upon there remain the same for the three-dimensional model: the sharing of the interface energy by the inner and outer liquid implies a decreasing of the time exponent - the pulsation $\omega$ as well as the growth factor $\gamma$.

\subsection{Comparison between linear models}

Finally, the main results obtained from the three-dimensional model presented above (free-motion frequencies of the bridge and the outer-fluid influence), can be compared with the results deduced from other formulations: infinite columns (Bauer 1982) and the one-dimensional model (\$3.2).

The infinite-surrounded-column model, derived from capillary-jet theory (Rayleigh 1945), towards which the Bauer model approaches when $\rho \rightarrow 0$, leads to the following expression:

$$
\omega^{2}=\frac{l_{n}\left(l_{n}^{2}-1\right) I_{1}\left(l_{n}\right)}{I_{0}\left(l_{n}\right) S_{n}}
$$

where $n$ is the number of half-cycles of the interface deformation, and $S_{n}$ is defined by (4.19), through which the influence of the bath parameters appears in the solution.

The linearized one-dimensional model for non-surrounded liquid bridges derived by Meseguer $(1983 a)$ gives an implicit relationship between slenderness and nonsurrounded liquid-bridge pulsation $\omega_{0}$ as follows:

$$
\sinh 2 \alpha \Lambda \sin 2 \beta \Lambda-\sqrt{ } 8 \omega_{0}\left(\cosh ^{2} \alpha \Lambda \cos 2 \beta \Lambda-\cos ^{2} \beta \Lambda\right)=0,
$$

where $2 \alpha^{2}=\left(1+8 \omega_{0}^{2}\right)^{\frac{1}{2}}-1$ and $\beta^{2}=1+\alpha^{2}$. The generalization of this model to the case including the bath has been made in $§ 3$ and consists of a timescaling

$$
\omega^{2}=\omega_{0}^{2}\left[1+\frac{\rho}{B^{2}-1}\right]^{-1}
$$

In order to appreciate the differences between results obtained from these models, the variation of the pulsation $\omega$ with $A$ in the case of a non-surrounded bridge has 


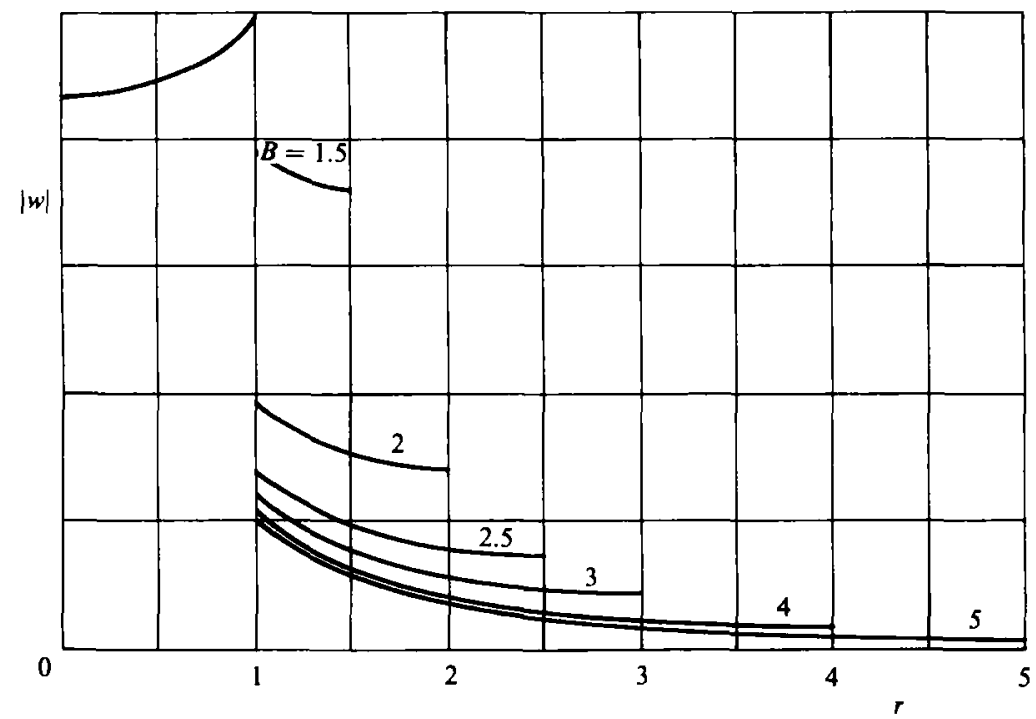

Fraure 12. Radial variation of axial-velocity modulus, $|w|$, in the mean section $(z=0)$, inside the liquid bridge $(r<1)$ and in the bath $(r>1)$ for several values of wall position $B$. First oscillation mode. Liquid bridge slenderness $A=2.9$.

been plotted in figure 6 , where it can be seen that the one-dimensional and three-dimensional models are in a good agreement within the range of applicability of the first model $\Lambda / n>1$. The results from Bauer, although they show the same behaviour as those from the three-dimensional model, are quite different in magnitude, because the infinite-column model satisfies the conditions for non-anchored liquid bridges, which are less restrictive than conditions for anchored configurations.

The influence on the pulsation $\omega$ of the parameters $A$ and $B$, for two bridges with large and small slenderness, is shown in figure 11. Predictions from the three models agree quite closely for large values of $\Lambda$ and $\rho \sim 1$, differences growing as $\Lambda$ decreases and $\rho$ increases. It can also be appreciated that, while in the three-dimensional and infinite-column models the bath influence on $\omega$ remains constant for large values of $B$, in the one-dimensional model $\omega$ tends to $\omega_{0}$ as $B$ increases, because in this model the bath effect disappears, since both the axial velocity and the kinetic energy absorbed by the bath decrease in the form $1 /\left(B^{2}-1\right)$. As shown in figure 12, in the three-dimensional model, even if $B$ is large, a region of the bath near the bridge is always in motion. The same figure shows the limit for the bath dimensions that should be satisfied for the one-dimensional model to be applicable; if $B$ is small the radial variations of axial velocity will also be small, but if $B$ increases they will become of the same order as the axial velocity, and the one-dimensional hypothesis will lose its basis.

\section{Experimental study}

The results derived from the linear models presented in $\$ 4$ can be experimentally checked on Earth by using a simulated microgravity technique. This is possible because bath influence is taken into account in these models. Amongst theoretical results, the dependence of the eigenfrequencies of the surrounded liquid bridge on the slenderness has been chosen to be experimentally studied. 


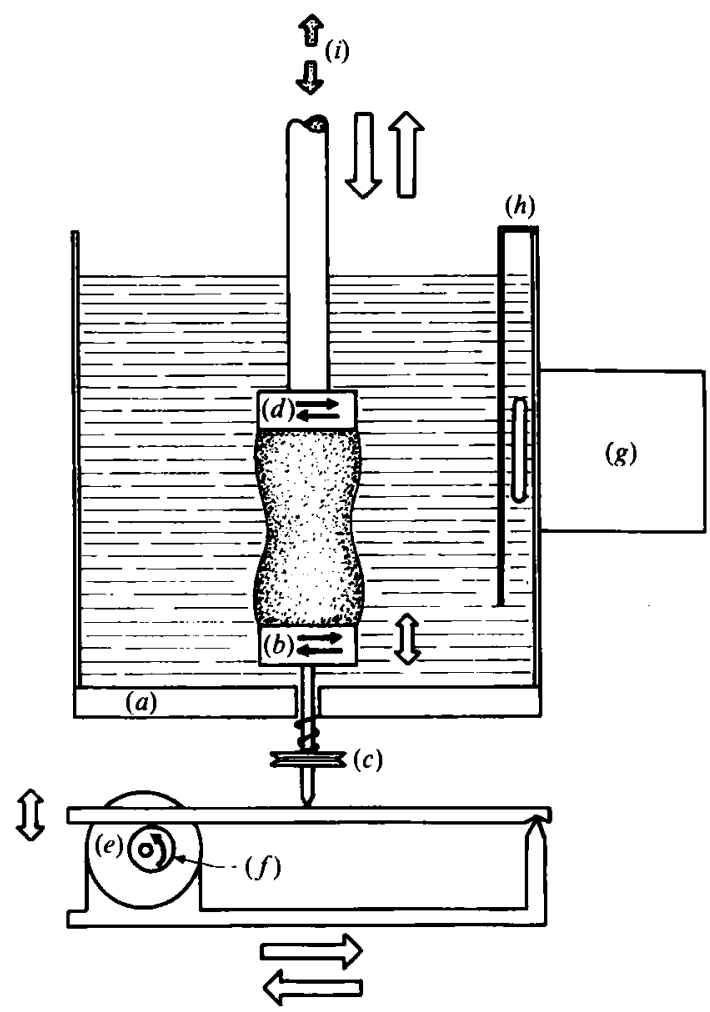

Figure 13. Experimental arrangement: $(a)$ tank; $(b)$ exciting disk; $(c)$ lower-disk turning pulley; $(d)$ injection disk; $(e)$ motor; $(f)$ eccentric; $(g)$ mixer; $(h)$ mixer shield; $(i)$ injection duct.

The method followed to find out the resonant frequencies consisted of determining the amplitude response of the interface deformation as a function of the oscillation frequency of one of the supporting disks. For small-enough viscous effects it could be assumed that the eigenfrequencies of the bridge almost coincided with the resonant frequencies of the forced system, at which the deformation amplitude reaches a maximum. This method was employed to determine the resonant frequencies of both non-surrounded millimetric liquid bridges (Fowle, Wang \& Strong 1979; Elagin, Lebedev \& Tsmelev 1982) and drops anchored to a disk in a Plateau tank (Bisch, Lasek \& Rodot 1982).

\subsection{Apparatus description}

The experiments were carried out in the Plateau Tank Facility (PTF) described in Martinez \& Rivas (1982). The PTF had already been employed on previous occasions to perform experiments in simulated microgravity by use of the neutral-buoyancy technique (Sanz 1983; Sanz \& Martinez 1983; Meseguer \& Sanz 1985). The PTF allows the manipulation of liquid bridges, supplying the main movements that are needed to perform a wide variety of experiments. Thus it is possible to move the injection disk axially, rotate both disks and even to oscillate the lower disk, as can be seen in figure 13. The tank, which contained the bath for neutral buoyancy, was a box with rectangular cross-section, $140 \mathrm{~mm} \times 60 \mathrm{~mm}$ sides and $140 \mathrm{~mm}$ height, with glass walls and perspex bottom. The tank top was closed by a plastic sheet to reduce evaporation. 


\begin{tabular}{|c|c|c|c|c|c|c|}
\hline \multirow[b]{2}{*}{$\boldsymbol{A}$} & \multicolumn{2}{|c|}{$f_{\mathrm{Ri}}(\mathrm{Hz})$} & \multirow[b]{2}{*}{$A_{\mathrm{e}} \times 10^{3}(\mathrm{~m})$} & \multirow[b]{2}{*}{$T\left({ }^{\circ} \mathrm{C}\right)$} & \multirow[b]{2}{*}{$F R(\mathrm{~Hz})$} & \multirow[b]{2}{*}{$D(\min )$} \\
\hline & Mode 1 & Mode 2 & & & & \\
\hline 1.0 & 0.97 & - & 1.3 & 20.9 & $0.8-1.2$ & 13 \\
\hline 1.0 & 0.97 & - & 1.3 & 20.9 & $0.8-1.2$ & 13 \\
\hline 1.1 & 0.82 & - & 1.3 & 20.9 & $0.6-1.0$ & 14 \\
\hline 1.2 & 0.72 & 1.53 & 1.3 & 22.0 & $0.5-1.8$ & 32 \\
\hline 1.3 & 0.62 & - & 1.3 & 23.0 & $0.4-1.0$ & 16 \\
\hline 1.3 & 0.61 & - & 1.3 & 23.0 & $0.4-1.0$ & 16 \\
\hline 1.5 & 0.45 & 1.06 & 1.3 & 22.0 & $0.4-1.2$ & 38 \\
\hline 1.67 & 0.36 & 0.88 & 1.3 & 22.0 & $0.3-1.2$ & 34 \\
\hline 1.8 & 0.30 & 0.75 & 1.3 & 24.0 & $0.1-1.6+$ & 48 \\
\hline 2.0 & - & 0.62 & 1.3 & 20.8 & $0.5-0.8$ & 15 \\
\hline 2.1 & - & 0.59 & 1.3 & 20.8 & $0.5-0.8$ & 15 \\
\hline 1.2 & 0.75 & 1.55 & 2.3 & 20.5 & $0.6-1.7$ & 37 \\
\hline 1.5 & 0.47 & 1.07 & 2.3 & 22.5 & $0.4-1.1$ & 38 \\
\hline 1.8 & 0.31 & 0.76 & 2.3 & 25.0 & $0.2-0.9$ & 35 \\
\hline
\end{tabular}

Wetting conditions were controlled through surface treatment of the disk lateral faces with an antispread barrier (FC-721 from 3M). Disks, of $30 \mathrm{~mm}$ diameter, were made of perspex and painted black to enhance visualization. The upper disk, supported by the injection tube, had an injection hole of $4 \mathrm{~mm}$ in diameter. The lower disk was mounted in a pin connected to an oscillator as shown in figure 13. With this arrangement, oscillatory motions of frequencies ranging from 0.1 to $2( \pm 0.005) \mathrm{Hz}$ could be obtained.

The outer liquid was a 1:2 methanol-water mixture, previously prepared by agitation and a later rest, to remove most of the air bubbles produced in the solution process. The liquid for the bridge, a dimethyl silicone oil with $20 \times 10^{-6} \mathrm{~m}^{2} \mathrm{~s}^{-1}$ viscosity, (trend-labelled DMS 20, from Rhone-Poulenc 1978) was coloured with yellow aniline dye to enhance visualization. Although the measured density of both liquids, the methanol-water mixture and the DMS 20 , was $\rho^{0}=\rho^{1}=954 \pm 0.5 \mathrm{~kg} \mathrm{~m}^{-3}$, the residual imbalance led to the cylindrical liquid bridges not having the necessary slenderness. Therefore a precise density matching was carried out before every experiment by adding either methanol or water to the bath. To this end the liquid bridge was used as a density indicator (Sanz \& Martinez 1983).

The management of the liquid for the bridge was performed with the aid of a syringe calibrated against a $0.2 \mathrm{~cm}^{3}$ graded burette. A magnetic stirrer was placed close to one of the lateral walls to homogenize concentration and temperature. The liquid bridge was protected against crossflow by placing a shield near the turning piece of the stirrer.

As a temperature controlled bath was not available, measurements of the temperature (as shown in table 2) were made during experiments with the aim of evaluating the results obtained; although the temperatures at which experiments were performed ranged between 20 and $25^{\circ} \mathrm{C}$, in each sequence this variation was less than $0.1^{\circ} \mathrm{C}$.

The visualization system consisted of a stroboscopic lamp, a heat filter, a 
0.18
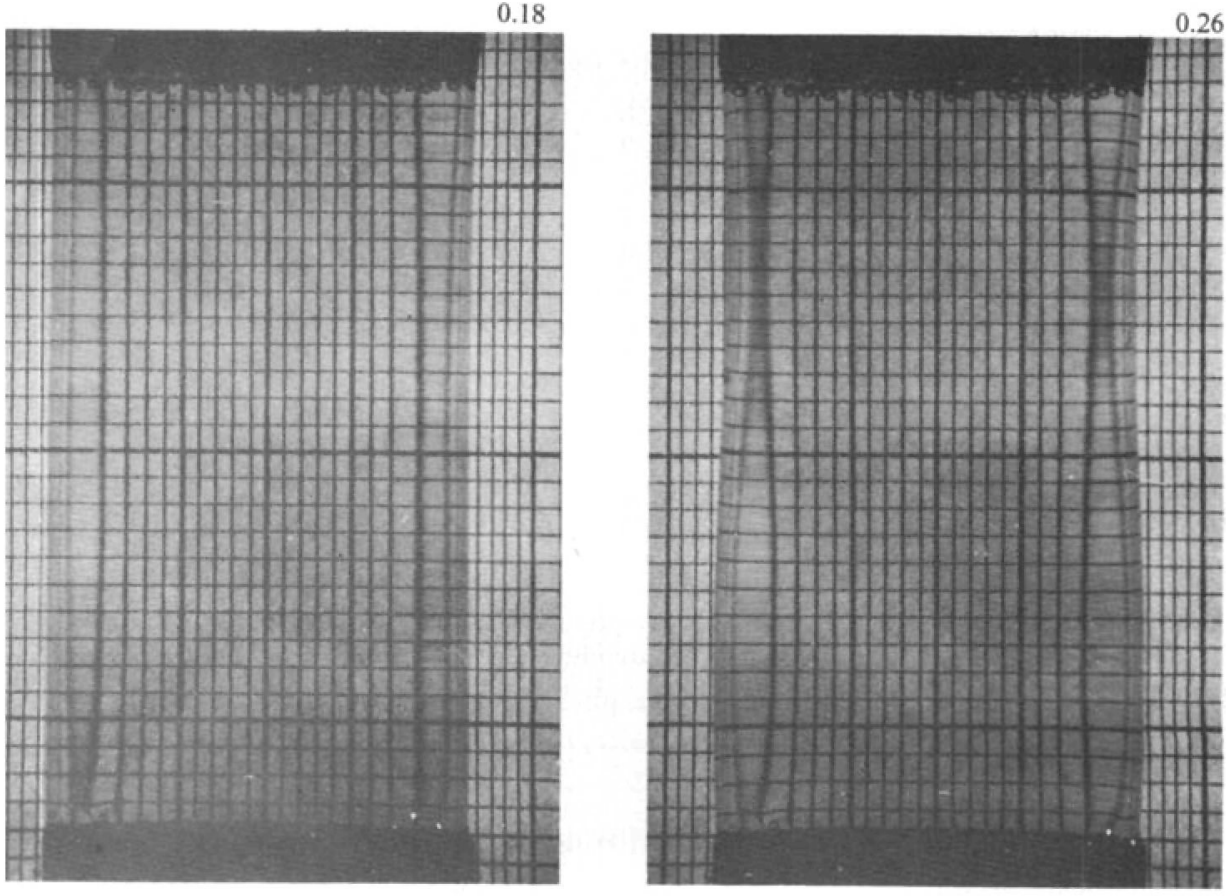

0.28

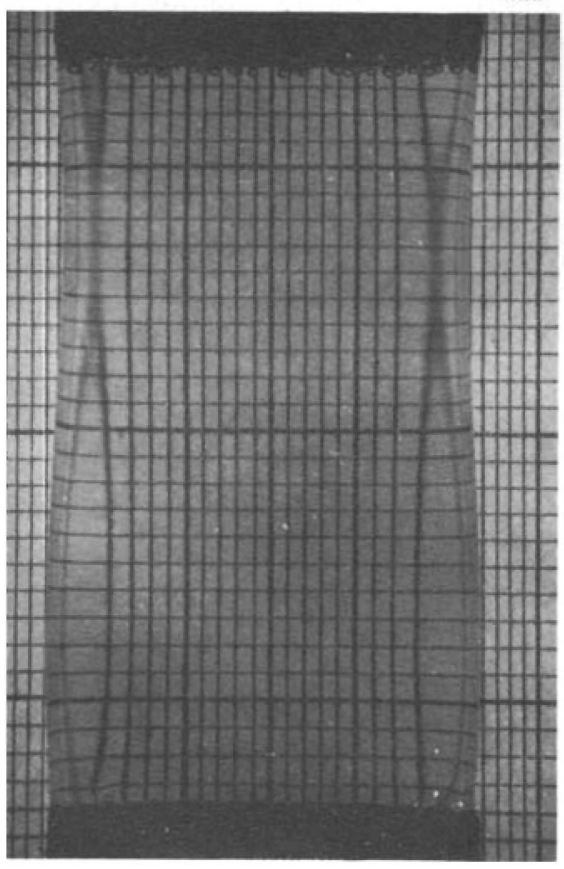

0.30

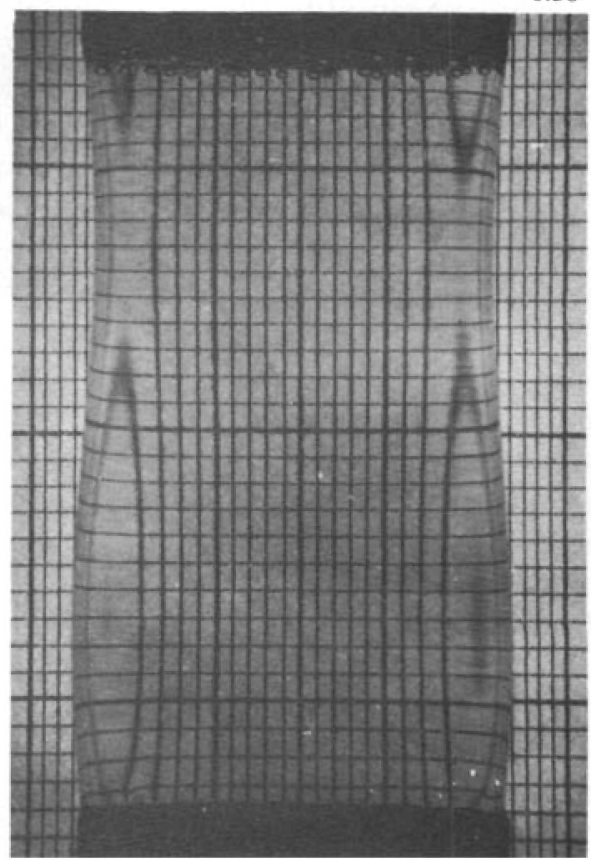

Figure 14. Part 1. For caption see p. 131. 
0.33

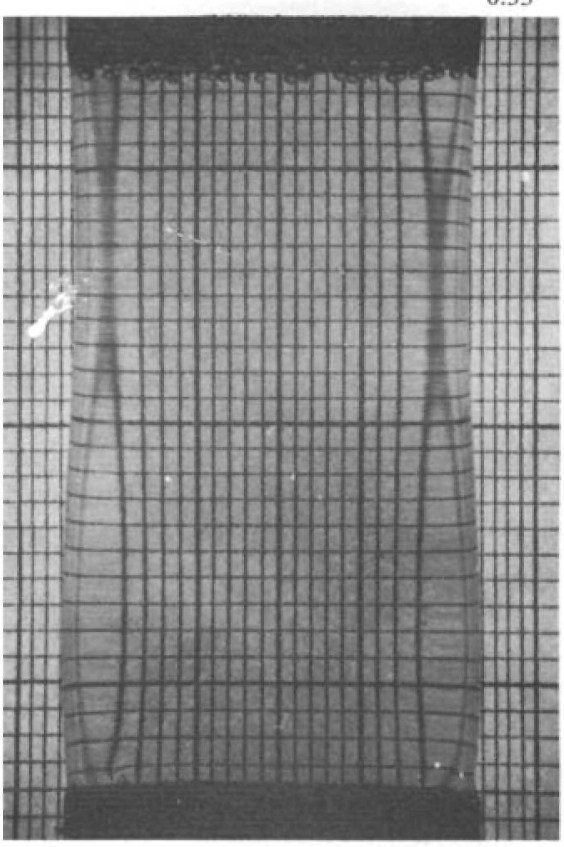

0.71

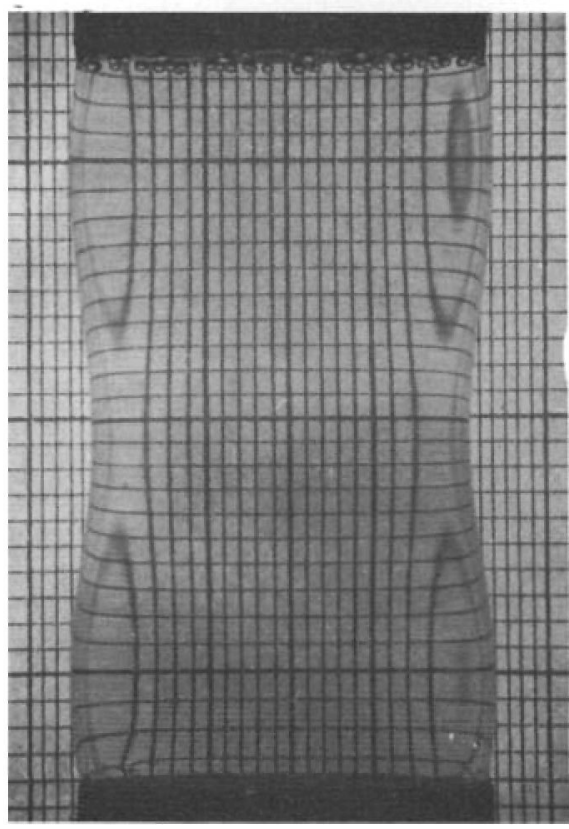

0.46

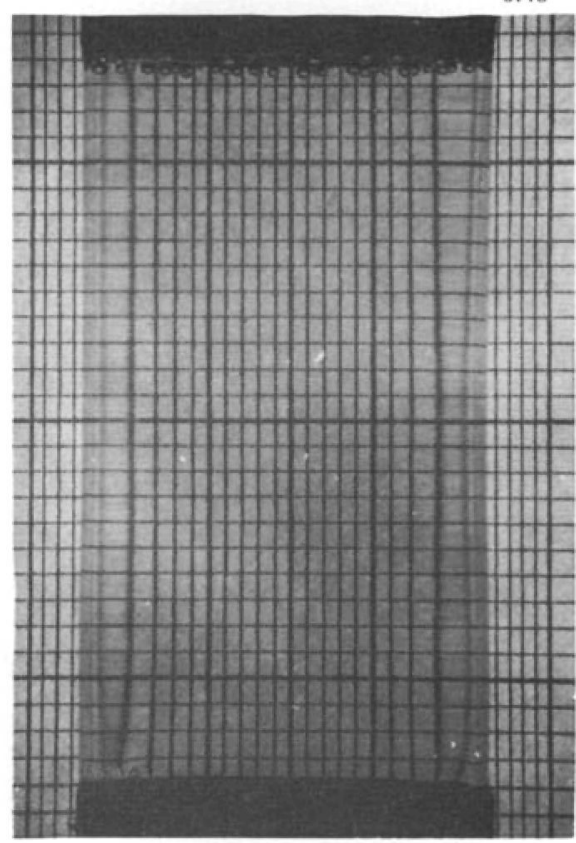

0.75

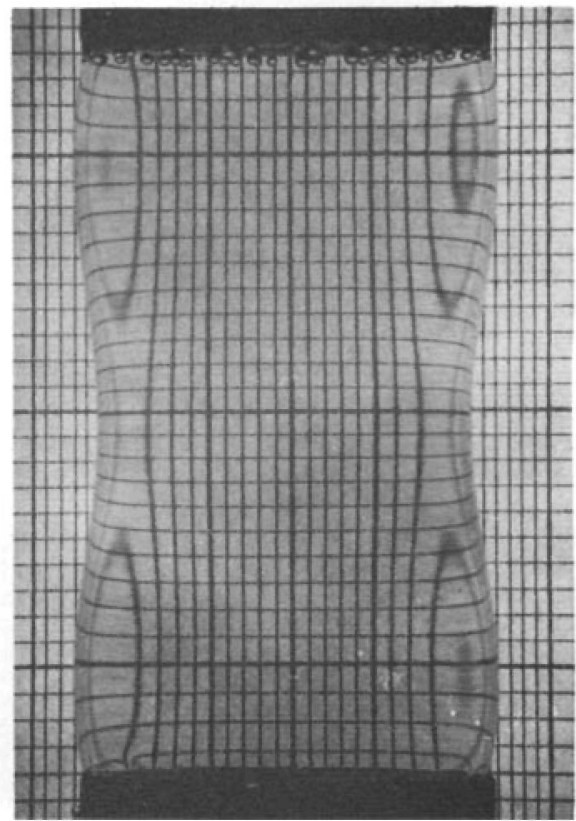

Figure 14. Part 2. For caption see p. 131. 

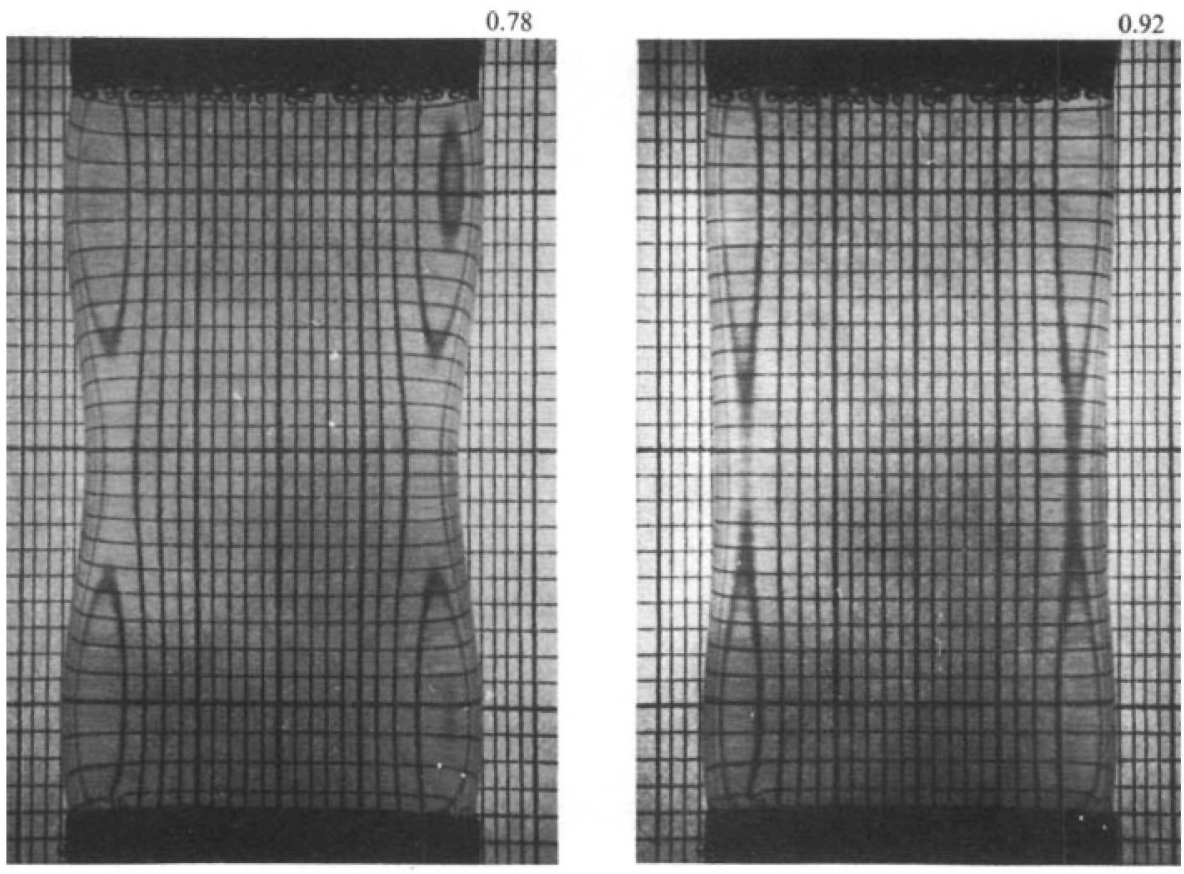

1.17
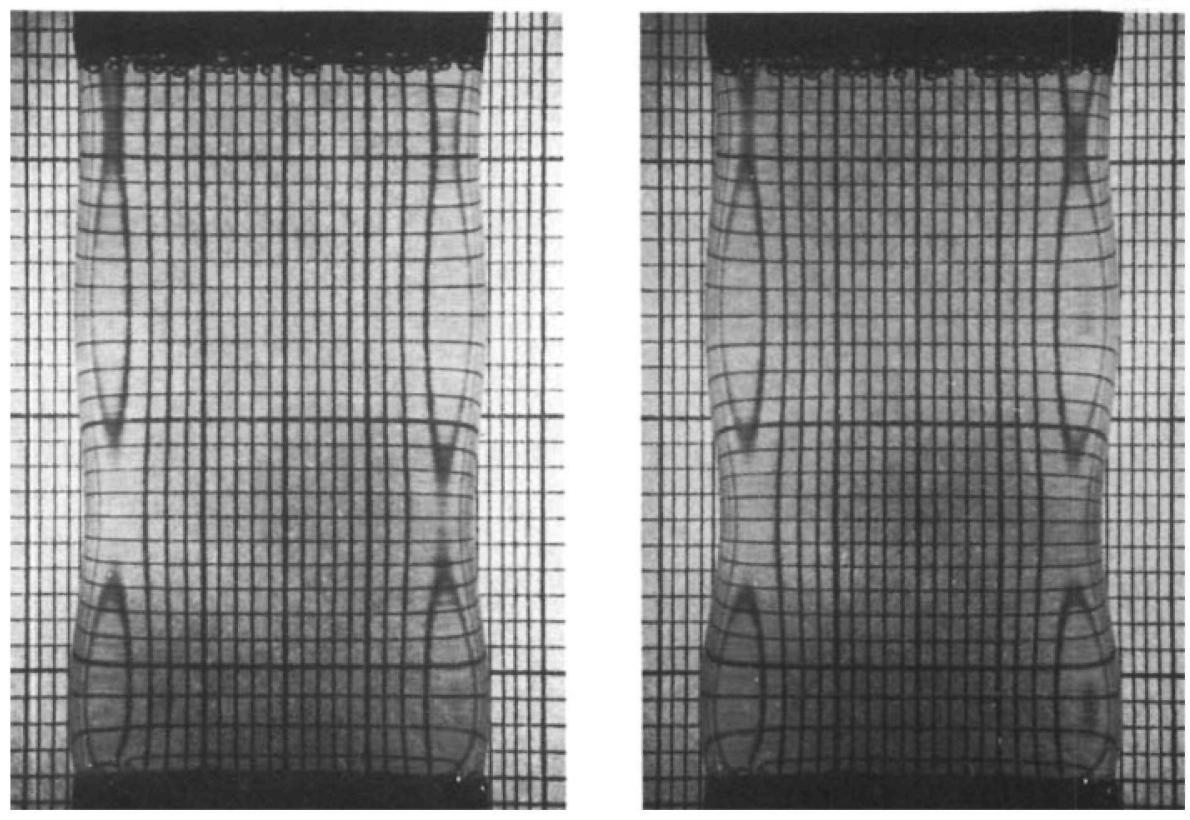

Figure 14. Part 3. For caption see opposite. 


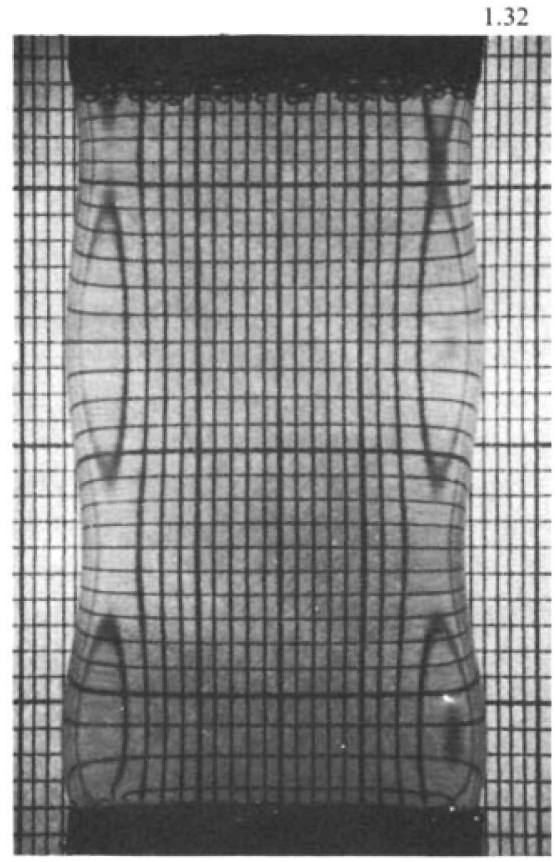

1.38
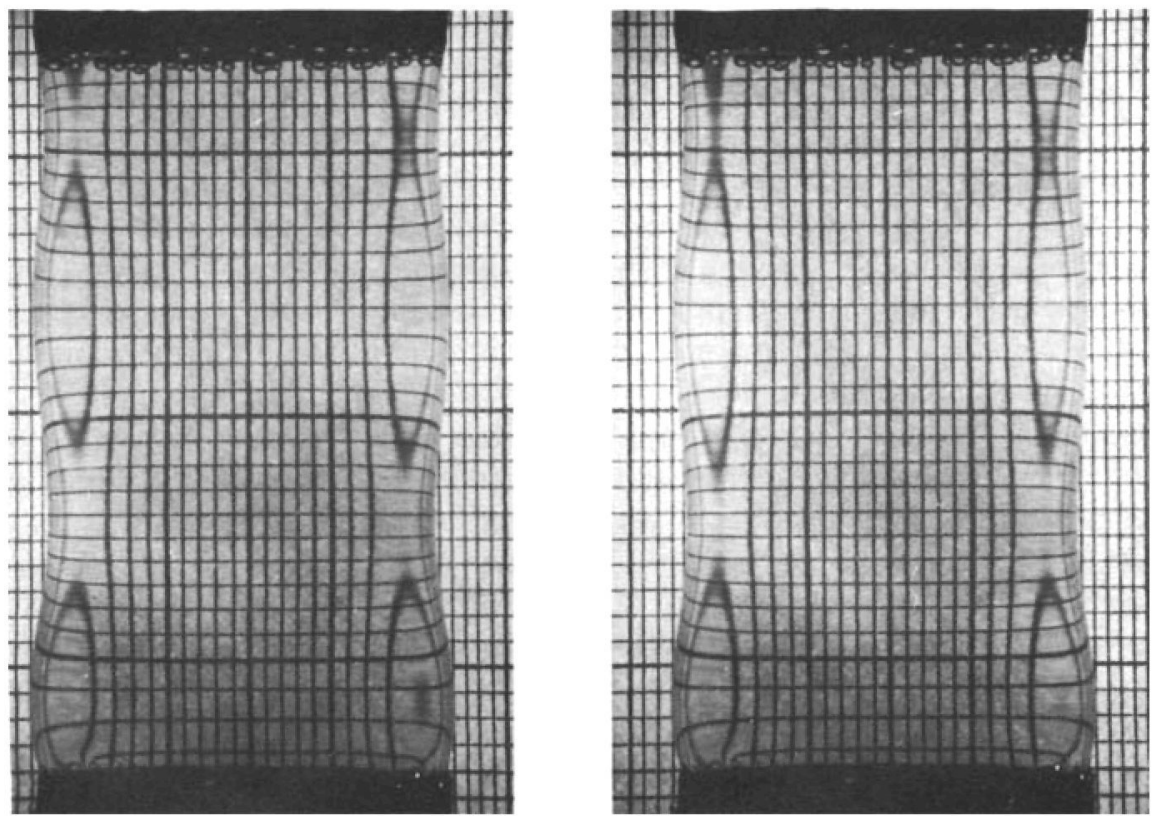

Figure 14. Selected photographs of an oscillation-scanning sequence. Liquid bridge of slenderness $\Lambda=1.8$ surrounded by a density-matched liquid. Numbers show the exciting frequency imposed $(\mathrm{Hz})$. First three resonant frequencies determined experimentally are $0.30,0.75$ and $1.29 \mathrm{~Hz}$ respectively. 
background grid and a camera. A $3 \times 10^{-6} \mathrm{~s}$ flash-duration stroboscopic lamp (STROBOTAC $1531 \mathrm{~A}$, General Radio Company), placed some $10 \mathrm{~cm}$ behind the rear tank wall, was synchronized with the disk oscillatory motion. The heat filter, consisting of a $1 \mathrm{~cm}$ thick water sheet, was placed between the lamp and the tank to reduce bath heating due to the illumination. A background grid, fixed to the tank rear pane, established a diffuse illumination and supplied reference lines for interfacedeformation measurements. Furthermore, the optical effect produced at the interface, due to refractive-index differences between outer and inner liquids, helped during experimentation to visualize interface deformations. A $35 \mathrm{~mm}$ camera was placed some $50 \mathrm{~cm}$ in front of the tank, with an image-object scale of some 1:2. The apparatus was placed inside a black room in order to avoid spurious illumination.

As mentioned above, it was necessary to perform a precise density matching before each experiment, because very small density differences between inner and outer liquids caused appreciable interface deformations. If the tank had not been sealed completely the differences in evaporation rate between alcohol and water would give rise to a variation of the bath density with time, establishing a maximum allowable experimentation period based on the limitation of interface deformations due to growing hydrostatic effects. These deformations were always kept smaller than those involved in oscillations. Details can be found in Sanz (1983).

\subsection{Methods and results}

Once a liquid bridge of the required length was obtained, the oscillator was started up at a fixed frequency. After waiting some time to avoid transients and to obtain a regular oscillation, the camera shutter was opened and remained so till the film was sufficiently exposed by the light of the stroboscopic lamp. Depending on the oscillation frequency, between 8 and 16 flashes were needed.

This process was repeated at constant liquid-bridge length, for slightly increased values of the imposed oscillation frequency. A selection of the photographs obtained in a typical scanning is shown in figure 14, where the first three modes of the interface deformation can be appreciated. In figure 15 the interface-deformation amplitudes measured from the images taken are plotted against the excitation frequency, in experiments performed with liquid bridges of several slendernesses. The maxima observed, sharp for first and smoother for second modes, are sufficient to deduce the corresponding resonant frequencies with an error of $\pm 0.02 \mathrm{~Hz}$.

The value of the deformation amplitude is taken as the mean of the maximum and minimum deformations measured on each image. To perform these measurements the image contained on the $35 \mathrm{~mm}$ film was enlarged some seven times with respect to the object. At the same axial sections, together with the maximum and minimum deformations, the distance between reference lines of the background grid was measured to obtain amplitude data and to perform the geometrical corrections needed owing to the optical arrangement. The errors in the deformations are of $\pm 0.08 \mathrm{~mm}$ at the object plane.

The existence of $\frac{1}{2} \pi$ delay between the excitation and the response of the liquid bridge was realized during the synchronization process. It could be explained in the following way: as can be deduced from the study of the problem using the three-dimensional inviscid model, maximum interface deformations should coincide with the moments at which the velocity, particularly at the disk, is null, which happen at the extreme positions of the oscillation travel. However, in experiments, the maximum deformations arose close to the mean point of the disk travel, giving rise 

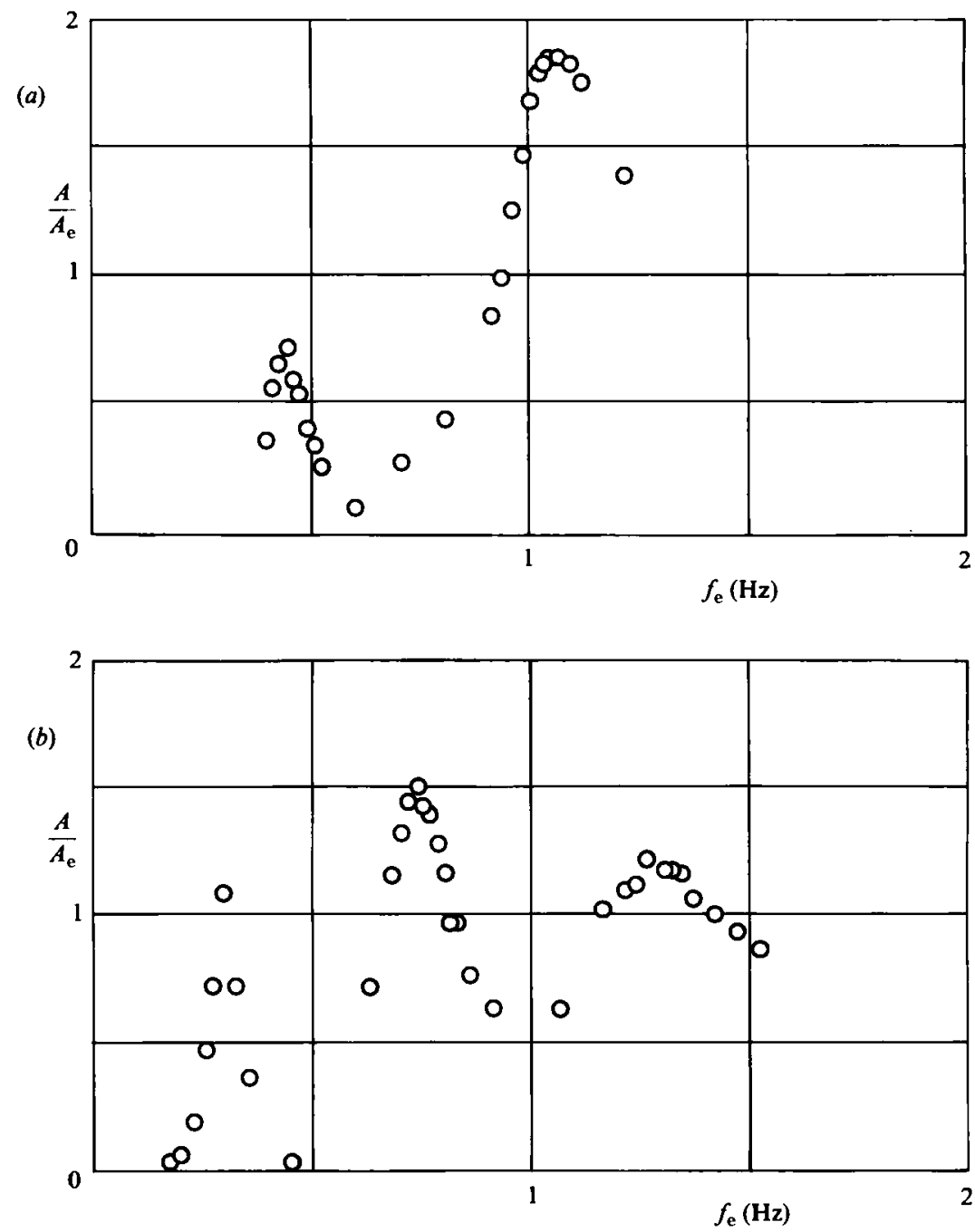

Figure 15. Ratio of deformation amplitude $A$ to excitation amplitude $A_{\mathrm{e}}$ versus excitation frequency $f_{\mathrm{e}}$ of several liquid bridges surrounded by a density-matched liquid: (a) $\Lambda=1.5$, $A_{\mathrm{e}}=1.3 \mathrm{~mm} ;(b) A=1.8, A_{\mathrm{e}}=1.3 \mathrm{~mm}$.

to this approximate $\frac{1}{2} \pi$ delay. The value of this delay is the same as that of the forced response of a linear oscillator with low damping. Excitative force and oscillation amplitude are in phase only if no damping exists. If dissipation is low, the resonant frequencies are close to the eigenfrequencies of the non-damped oscillator, and the delay at these frequencies is about $\frac{1}{2} \pi$.

With the aid of amplitude-versus-frequency plots (as in figure 15) the resonant frequencies were determined for several values of $\Lambda$ (table 2 ). The largest influence of the excitation amplitude on the variation of the deformation amplitude with the imposed frequency, for the values shown in table 2 , is of some $4 \%$ for the lowest slenderness $(\Lambda=1.2)$. So we consider as valid the values obtained with the smallest amplitude tried, $A_{\mathrm{e}}=1.3 \mathrm{~mm}$. This amplitude is the minimum advisable to obtain deformation measurements with a reasonable error. The maximum interface deformations pro- 


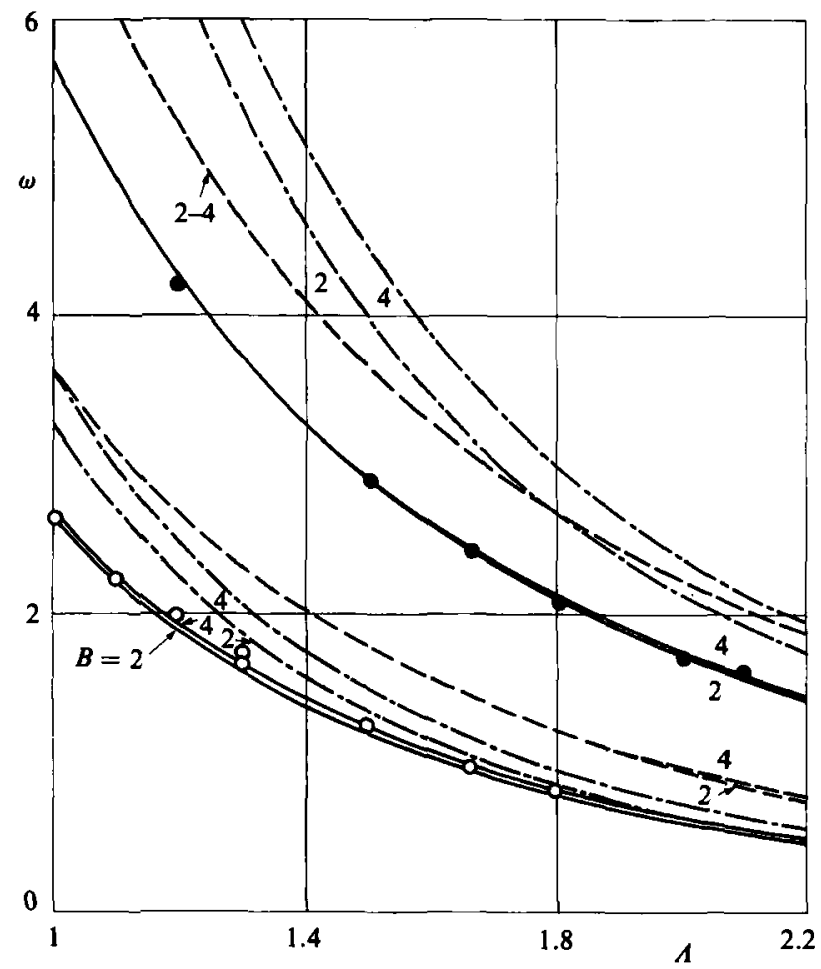

Fraure 16. Pulsation $\omega$ versus slenderness $\Lambda$ of a density-matched surrounded liquid bridge for several values of wall position $B$, obtained from linearized models: three-dimensional (solid line), infinite columns (dashed line) and one-dimensional (dot-dashed line). Experimental results of table 2, made dimensionless with (5.1): $\bigcirc$, first mode; $\bigcirc$, second mode.

duced by the excitation amplitude tried are of the order of $17 \%$ of the radius, so that the results obtained are appropriate for comparison with those from linearized theoretical models. Measurements have been reduced according to the expression

$$
\omega_{\mathrm{E} i}=2 \pi f_{\mathrm{R} i}\left(\frac{\rho^{1} R^{3}}{\sigma}\right)^{\frac{1}{2}}
$$

where $\omega_{\mathbf{E} i}$ is the experimental dimensionless pulsation and $f_{\mathrm{R} i}$ stands for the $i$ th resonant frequency measured. $\sigma$ is determined in the Appendix. Results are shown in figure 16. The experimental limit values of $A$ are established, at low frequencies, by the oscillator range and viscous effects (large $\Lambda$, small $\Omega$ ), and by amplitudemeasuring limitations at high frequencies (small $A$ ).

\subsection{Comparison between experiments and theory}

The experimental results have been compared with the following linear models: the infinite-column model (Bauer 1982), the one-dimensional model developed in $\$ 3.2$ and the three-dimensional model described in $\$ 4$, whose results in the experimental range are plotted in figure 16.

The first point is that experimental and theoretical configurations are not identical. In the latter the cross-section of the tank is a circle, while in the experimental set-up it is rectangular (walls placed at $B=2$ and $B=4.67$ respectively) owing to the optical arrangement. However, when the experiment was designed, it was considered that 


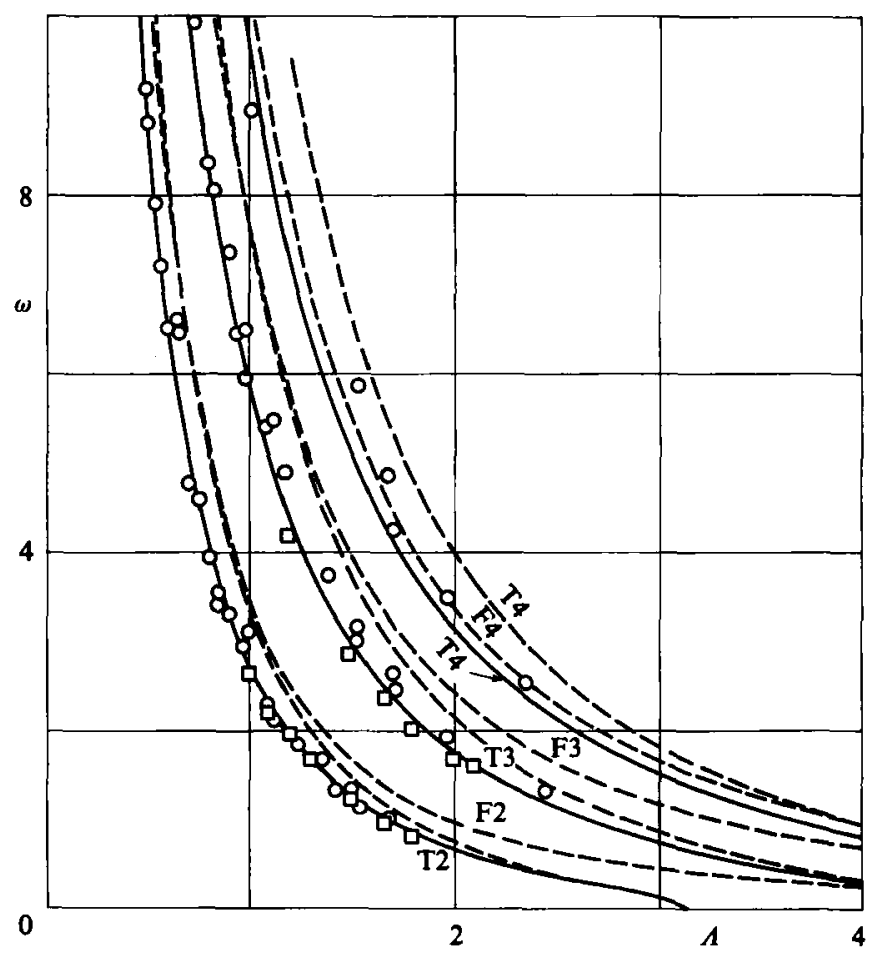

FIGURE 17. Variation of pulsation $\omega$ with slenderness $A$ in the first oscillation modes of liquid bridges: non-surrounded (dashed line), and surrounded by a density-matched liquid (solid line). The wall is placed at $B=4$. Results obtained from linearized models: F, Fowle et al. (1979); T, three-dimensional. Figures indicate the oscillation modes (number of half-waves in the interface deformation). Experimental results: $\mathcal{O}$, Fowle; $\square$, from table 2 and made dimensionless with $\sigma=17.0 \times 10^{-3} \mathrm{~N} \mathrm{~m}^{-1}$.

the rectangular shape would not be a problem, as far as predictions from the three-dimensional and Bauer's models are concerned. In these models the influence of the wall position remains constant for large values of $B$, as shown in figures 11 and 16. Another difference is the position of the bounding walls at $z= \pm A$. Because of physical constraints these walls were placed some $10 \mathrm{~mm}$ from the surface of the disks, instead of at the very surface. To take account of this modification in the theoretical formulation would be very difficult, so this could have been a source of discrepancy between theory and experiments. Nevertheless, such a discrepancy should be small since the kinetic energy transferred to this region of the bath is also small, owing to the proximity of the walls.

Experimental and theoretical results from Fowle et al. (1979), shown in figure 17, are treated separately, as they correspond to non-surrounded liquid bridges. The theoretical model of Fowle et al. yields the following expression :

$$
\omega^{2}=l_{n}^{3}\left\{\frac{I_{0}\left(l_{n}\right)}{I_{1}\left(l_{n}\right)}+\frac{l_{n}}{s}\left[\frac{I_{0}^{2}\left(l_{n}\right)}{I_{1}^{2}\left(l_{n}\right)}-1\right]\right\}^{-1},
$$

where $s=2$ (or 1 ) for symmetric (or antisymmetric) deformations, and $l_{n}=n \pi / 2 \Lambda$. To obtain (5.2) some assumptions, whose validity is not clear, are introduced in this model. As a consequence one of the main characteristics of the problem, the existence of the stability limit in the first mode at $\Lambda=\pi$ is not predicted. However, the 
behaviour of results from this model, for small values of $A$ and the first two oscillation modes, seems to be in agreement with those from the three-dimensional model. Surprisingly, experimental results from Fowle et al. are in agreement with the prediction from the three-dimensional model for the case of a liquid bridge surrounded by a density-matched bath, at least for the first modes and at low frequencies.

\section{Conclusions}

The main effects due to the presence of an outer liquid in both breaking process and oscillation motions of liquid bridges have been considered. Theoretical support for experimental results on liquid-bridge dynamics from using the Plateau-tank technique has been developed, valid within the range of applicability of the studies here performed. Additional efforts should be devoted to the evaluation of the influence of the outer bath in experiments in which rotation of the disks is involved.

The nonlinear model developed in this paper allows us to perform the computation of breaking processes. Amongst other results, it can be pointed out that bath effects slightly modify partial breaking volumes, in current experiments. Thus partial volumes are a valuable candidate for experimental checking of the ODIS model both under simulated microgravity conditions (as in Meseguer \& Sanz 1985) and in Space. Experimental checking of the temporal evolution is more complex owing to the difficulty of imposing on a liquid bridge the initial perturbations more appropriate from a numerical point of view, initial conditions having a strong influence on breaking times. The validity of the one-dimensional model is restricted by (3.2) and (3.3).

A double approach has been followed to study oscillatory motions of surrounded liquid bridges. First, a linear three-dimensional model of the problem, accounting for the outer liquid, has been developed. As a consequence of the fact that the influence of wall position remains almost constant for large values of the wall-liquid-bridge distance, the tank shape would not have significant influence if its lateral walls are far enough apart from the liquid-bridge axis. This conclusion allows simplification of the experimental set-up.

In a second approach, an experimental method to measure the resonant frequencies of liquid bridges has been developed. The results obtained have been used for evaluating several theoretical models, although a relaxed shape for the tank is assumed. The three-dimensional model, which retains the appropriate boundary conditions and minimum simplifications, offers better agreement with experiments in the range checked. The results from the one-dimensional model in the first mode coincide quite well for $\Lambda>2$ with those from the three-dimensional model, as is shown in figures 6 and 16. This bound on $A$ can be considered as a validity limit of the one-dimensional model in studying linear oscillations. A similar value is obtained in the case of breaking processes (Meseguer et al. 1983).

As an additional result, a method based on measuring the resonant frequencies of a liquid bridge seems to be useful for determination of surface tension in a microgravity environment, in which classical methods based on gravity effects are not applicable.

To conclude, some considerations on the viscosity effects should be made. The usual criterion to consider unsteady flows as inviscid is that the ratio of inertial to viscous terms in the Navier-Stokes equations, $S t R e=\lambda^{2} \Omega / \nu \gg 1$, where $S t$ and $R e$ are the Strouhal and Reynolds number, $\lambda$ is a characteristic length (the length of the bridge in a breaking process, or the wavelength in oscillations), $\Omega$ is the dimensional 
pulsation or growth factor and $\nu$ is the kinematic viscosity. In the case of oscillation (first mode, $\Lambda \approx \pi$ )

$$
\Omega^{2} \approx\left(\frac{\rho^{1} R^{3}}{\sigma}\right)^{-1}(\Lambda-\pi)\left[1+\frac{\rho}{B^{2}-1}\right]^{-1}
$$

In the experiments reported here the lowest value of $S t R e=1.5 \times 10^{2}(\Lambda=1.2$, $\Omega=9.6 \mathrm{rad} / \mathrm{s}, \lambda=18 \mathrm{~cm})$. In a general case, if $\Omega \rightarrow 0(\Lambda \rightarrow \pi, B \rightarrow 1$ or $\rho \rightarrow \infty)$, this number is greatly reduced, and the assumption of inviscid flow would no longer be valid.

This work has been supported by the Spanish National Commission for Space Research (CONIE) under a contract with the Polytechnic University of Madrid (UPM). I wish to thank Professor I. Da-Riva, Dr J. M. Vega and Dr J. Meseguer for helpful discussions.

\section{Appendix. Determination of the interface tension between density-matched liquids}

To make dimensionless the experimental results obtained, it is necessary to know the value of the interface tension $\sigma$ (5.1). Owing to the lack of data in the literature on the interface tension between the liquids used, it has to be measured.

There are several methods to determine experimentally the value of $\sigma$ in the case of liquid interfaces. The pendant-drop method can be employed for liquid-liquid interfaces only if the liquids have appreciable differences in density. In experiments in a Plateau tank, some authors use this method aided by interpolation (Carruthers \& Grasso 1972; Tagg et al. 1980; Bisch et al. 1982). If the viscosity is high this interpolation is not very reliable, as shown by Tagg et al. and Bisch et al. Another method, developed by Princen; Zia \& Mason (1967), is based on the measurement of the interface deformations of liquids or air drops submerged in another liquid; the deformation is produced by the rotation of the whole configuration. This method is particularly useful for viscous systems, but it also needs a density difference to cause deformations.

A method that in principle does not require such density differences and is therefore applicable to density-matched liquids (based on the measurement of the wavelength of capillary waves induced at an interface) has been developed by Milgram \& Bradley (1971) and employed with the couple mineral-oil-water (whose densities are quite different). Results obtained show values of $\sigma$ with errors of some $10 \%$, caused by visualization problems which have an important influence on the wavelengthmeasurement process. These problems grow as the density difference decreases (Meseguer 1983, private communication).

In the experimental study presented here, $\sigma$ was determined by using a variation of the bubble method. A drop of oil was formed at the tip of a capillary tube, submerged in the outer liquid. The pressure inside the drop was controlled by changing the height of a burette connected to the capillary. The height was measured with the micrometer screw, which positioned the burette, and the size of the cap at the tip with a microscope. Let $l_{\mathrm{d}}$ be the cap size and $h$ the difference in level between the liquid of tank and the burette, made dimensionless with the capillary radius $R_{\mathrm{c}}=0.55 \mathrm{~mm}$ (figure 18). The relationship between $l_{\mathrm{d}}$ and $h$ is

$$
y \equiv \frac{l_{\mathrm{d}}}{1+l_{\mathrm{d}}^{2}}=\frac{1}{4} B o_{\mathrm{c}} h
$$




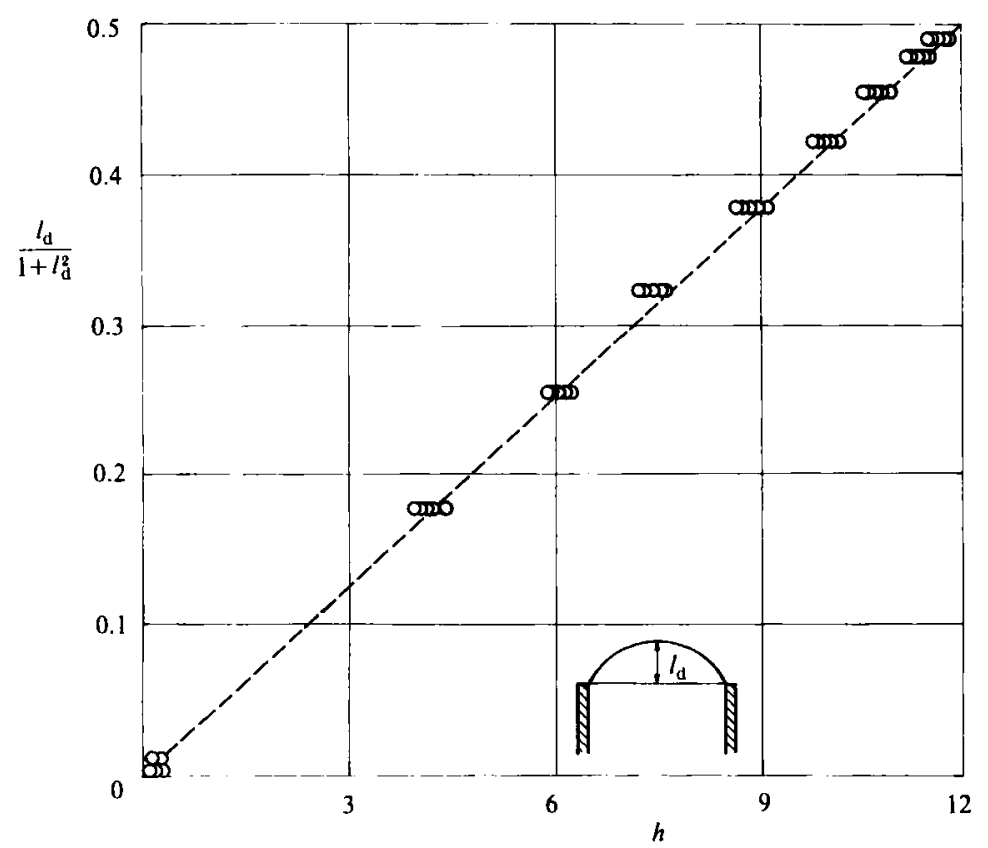

Figure 18. Interface-tension measurements. Correlation between ex perimental data and theoretical predictions (A 1). $l_{\mathrm{d}}$, cap height; $h$, difference in level between the liquid outside and inside the capillary. Lengths are made dimensionless with the capillary radius. Dashed line, linear regression.

where $B o_{\mathrm{c}}=\rho^{\mathrm{i}} g R_{\mathrm{c}}^{2} / \sigma$ is the Bond number associated with the capillary phenomena. Experimental results are shown in figure 18, together with a linear regression between $y$ and $h$, from which the following values can be obtained:

$$
B o_{\mathrm{c}}=(16.7 \pm 0.1) \times 10^{-2}, \quad \sigma=(17.0 \pm 0.1) \times 10^{-3} \mathrm{~N} \mathrm{~m}^{-1}
$$

The lack of data concerning interface tension does not allow an extensive comparative study. The only data that have been found are related to interfaces between density-matched methanol-water mixtures and DMS 5 and DMS 10 (Bisch et al. 1982), whose values are $\sigma=7 \times 10^{-3} \mathrm{~N} \mathrm{~m}^{-1}, \sigma=14 \times 10^{-3} \mathrm{~N} \mathrm{~m}^{-1}$ respectively, obtained by the aforementioned pendant-drop and interpolation method. The value obtained by using the bubble method is larger than that corresponding to DMS 10 , which is in agreement with the correlation between viscosity and interface tension shown by the data from Bisch et al.

\section{REF ERENCES}

BAUER, H. F. 1982 Coupled oscillations of a solidly rotating liquid bridge. Acta Astronaut. 9, $547-563$.

Bisch, C., LASEK, A. \& RoDot, H. 1982 Hydrodynamic behaviour of spherical semi-free liquid volumes in simulated weightlessness. J. Méc. Theor. Appl. 1, 165-183.

Carruthers, J. R. \& Grasso, M. 1972 The stabilities of floating liquid zones in simulated zero gravity. J. Cryst. Growth 13/14, 611-614.

Coriell, S. R., Hardy, S. C. \& Cordes, M. R. 1977 Stability of liquid zones. J. Coll. Interface Sci. 60, 126-136. 
Da Riva, I. \& Alvarez Pereira, E. 1982 A regular perturbation approach to surface tension driven flows. Acta Astronaut. 9, 217-224.

Da Riva, I. \& Mesequer, J. 1978 On the structure of the floating zone in melting. Acta Astronaut. S, 637-653.

Elagin, M. P., Lebedev, A. P. \& Tsmelev, A. V. 1982 Laboratory modeling of the stability and dynamics of free liquid zones. In Hydromechanics and Heat and Mass Transfer in Zero-Gravity (in Russian), pp. 24-33. Nauka.

Erle, M. A., Gillette, R. D. \& Dyson, D. C. 1970 Stability of interfaces of revolution with constant surface tension. The case of catenoid. Chem. Engng J. 1, 97-109.

Fowle, A. A., Wang, C. A. \& Strona, P. F. 1979 Experiments on the stability of conical and cylindrical liquid columns at low Bond numbers. ADL Ref. C-82435, Arthur D. Little, Inc., Cambridge, Massachusetts.

Gillette, R. D. \& Dyson, R. C. 1971 Stability of fluid interfaces of revolution between equal solid circular plates. Chem. Engng J. 2, 44-54.

Harriot, G. M. \& Brown, R. A. 1983 Flow in a differentially rotated cylindrical drop at low Reynolds number. J. Fluid Mech. 126, 269-285.

Levich, V. G. 1962 Physicochemical Hydrodynamics, chap. XI, pp. 591-668. Prentice-Hall.

LEYbold-Heraeus, 1977 Floating Zone melting of refractory metal rods. 61S1.210e, LeyboldHeraeus GmbH \& Co. KG, Bonner Strasse 504, 0-5000 Köln 51.

Martinez, I. 1976 Floating zone under reduced gravity. Axisymmetric equilibrium shapes. In Material Sciences in Space ESA SP-114, pp. 277-282. Paris: ESA.

Martinez, I. 1978 Floating zones. Equilibrium shapes and stability criteria. In COSPAR: Space Research Vol. XVIII (ed. M. J. Rycroft \& A. C. Strickland), pp. 519-522. Pergamon.

Martinez, I. \& Rivas, D. 1982 Plateau Tank Facility for simulation of Spacelab experiments. Acta Astronaut. 9, 339-342.

Mason, G. C. 1970 An experimental determination of the stable length of cylindrical liquid bubbles. J. Coll. Interface Sci. 32, 172-176.

Mesequer, J. $1983 a$ The breaking of axisymmetric slender liquid bridges. J. Fluid Mech. 130, 123-151.

MEsEgUeR, J. $1983 b$ The influence of axial microgravity on the breakage of axisymmetric slender liquid bridges. J. Cryst. Growth 62, 577-586.

Meseguer, J.\& Sanz, A. 1985 Numerical and experimental study of the dynamics of axisymmetric slender liquid bridges. J. Fluid Mech. 153, 83-101.

Meseguer, J., Sanz, A. \& Rivas, D. 1983 The breaking of axisymmetric non-cylindrical liquid bridges. In Materials Sciences under Microgravity ESA SP-191, pp. 261-265. Paris: ESA.

Milgram, J. H. \& Bradley, R. G. 1971 The determination of the interfacial tension between two liquids. J. Fluid Mech. 50, 469.

Mitchell, A. R. \& Griffiths, D. F. 1980 The Finite Difference Method in Partial Differential Equations. Wiley.

Napolitano, L. G. 1978 Microgravitational fluid dynamics. In Proc. 2nd Levich Cong., Washington.

Phinney, R. E. 1973 Stability of a laminar viscous jet - the influence of an ambient gas. Phys. Fluids 16, 193-196.

Princen, H. M., ZiA, I. Y. \& Mason, S. G. 1967 Measurement of interfacial tension from the shape of a rotating drop. J. Coll. Interface Sci. 23, 99.

Provost, P. \& Joy al, M. 1972 Thermodynamique. Masson.

RAYLEIGH, LoRD 1945 The Theory of Sound, vol. 2. Dover.

Rhone-Poulenc 1978 Rhodorsil silicones. Ref. X-03-03b, Rhone-Poulenc Chimie Fine, Dept Silicones, Paris.

Rivas, D. \& Meseguer, J. 1984 One-dimensional, self-similar solution of the dynamics of axisymmetric slender liquid bridges. $J$. Fluid Mech. 138, 417.

Roache, P. J. 1972 Computational Fluid Dynamics. Hermosa.

SAnz, A. 1983 Comportamiento de las zonas liquidas flotantes en microgravedad simulada. Tesis doctoral, Universidad Politecnica de Madrid. 
Sanz, A. \& Martinez, I. 1983 Minimum volume for a liquid bridge between equal disks. J. Coll. Interface Sci. 93, 235-240.

Tage, R., Cammack, L., Cronquist, A. \& Wang, T. G. 1980 Rotating liquid drops: Plateau's experiment revisited. JPL 900-954, Jet Propulsion Laboratory, Caltech.

Tомотік A, S. 1935 On the instability of a cylindrical thread of a viscous liquid surrounded by another viscous fluid. Proc. $R$. Soc. Lond. A 150, 322-337. 